\title{
Quantitative Susceptibility Atlas Construction in Montreal Neurological Institute Space: Towards Histological-consistent Iron-rich Deep Brain Nucleus Sub-region Identification
}

Chenyu He ( $\sim$ hechy1@shanghaitech.edu.cn )

ShanghaiTech University https://orcid.org/0000-0003-2730-4437

\section{Xiaojun Guan}

Zhejiang University School of Medicine Second Affiliated Hospital

Weimin Zhang

ShanghaiTech University

Jun Li

ShanghaiTech University

Chunlei Liu

University of California Berkeley

Hongjiang Wei

Shanghai Jiao Tong University

Xiaojun Xu

Zhejiang University School of Medicine Second Affiliated Hospital

Yuyao Zhang

ShanghaiTech University

\section{Research Article}

Keywords: deep brain nuclei, quantitative susceptibility mapping, quantitative susceptibility atlas, multimodal atlases, basal ganglia, thalamus, parcellation

Posted Date: November 10th, 2021

DOI: https://doi.org/10.21203/rs.3.rs-994193/v1

License: (c) (i) This work is licensed under a Creative Commons Attribution 4.0 International License.

Read Full License 


\section{Quantitative susceptibility atlas construction in Montreal Neurological Institute space: towards histological-consistent iron-rich deep brain nucleus sub-region identification}

Chenyu $\mathrm{He}^{1 \dagger}$, Xiaojun Guan ${ }^{2 \dagger}$, Weimin Zhang ${ }^{1}$, Jun $\mathrm{Li}^{1}$, Chunlei $\mathrm{Liu}^{3}$, Hongjiang Wei ${ }^{4,5}$, Xiaojun $\mathrm{Xu}^{2^{*}}$ and Yuyao Zhang ${ }^{1,6^{*}}$

${ }^{1}$ School of Information Science and Technology, ShanghaiTech University, 393 Huaxia Road, Shanghai, 201210, China. ${ }^{2}$ Department of Radiology of The Second Affiliated Hospital, Zhejiang University School of Medicine, 88 Jiefang Road, Hangzhou, 310009, Zhejiang, China.

${ }^{3}$ Electrical Engineering and Computer Science, University of California at Berkeley, Berkeley, 94720, CA, United States.

${ }^{4}$ School of Biomedical Engineering, Shanghai Jiao Tong University, 800 Dongchuan Road, Shanghai, 200030, China. ${ }^{5}$ Institute of Medical Robotics, Shanghai Jiao Tong University, 800 Dongchuan Road, Shanghai, 200030, China. ${ }^{6}$ Shanghai Engineering Research Center of Intelligent Vision and Imaging, ShanghaiTech University, 393 Huaxia Road, Shanghai, 201210, China.

*Corresponding author(s). E-mail(s): xxjmailbox@zju.edu.cn; zhangyy8@shanghaitech.edu.cn;

Contributing authors: hechy@shanghaitech.edu.cn; xiaojunguan1102@zju.edu.cn; zhangwm@shanghaitech.edu.cn; lijun4@shanghaitech.edu.cn; chunlei.liu@berkeley.edu; hongjiang.wei@sjtu.edu.cn;

†These authors contributed equally to this work. 


\begin{abstract}
Iron-rich deep brain nuclei (DBN) of the human brain are involved in various motoric, emotional and cognitive brain functions. The abnormal iron alterations in the DBN are closely associated with multiple neurological and psychiatric diseases. Quantitative susceptibility mapping (QSM) provides the spatial distribution of tissue magnetic susceptibility in the human brain. Compared to traditional structural imaging, QSM has superiority for imaging the iron-rich DBN owing to the susceptibility difference existing between brain tissues. In this study, we construct a Montreal Neurological Institute (MNI) space unbiased QSM human brain atlas via group-wise registration from 100 healthy subjects aged 19-29 years. The atlas construction process is guided by hybrid images that fused from multi-modal Magnetic Resonance Images (MRI), thus named as Multi-modal-fused magnetic Susceptibility (MuSus-100) atlas. The high-quality susceptibility atlas provides extraordinary image contrast between iron-rich DBN with their surroundings. Parcellation maps of DBN and their sub-regions that are highly related to neurological and psychiatric pathology are then manually labeled based on the atlas set with the assistance of an image border-enhancement process. Especially, the bilateral thalamus is delineated into 64 detailed sub-regions referring to the Schaltenbrand and Wahren stereotactic atlas. To our best knowledge, the histological-consistent thalamic nucleus parcellation map is well defined for the first time in MNI space. Comparing with existing atlases emphasized on DBN parcellation, the newly proposed atlas outperforms on atlas-guided individual brain image DBN segmentation accuracy and robustness. Moreover, we apply the proposed DBN parcellation map to conduct detailed identification of the pathologyrelated iron content alterations in subcortical nuclei for Parkinson Disease (PD) patients. We envision that the MuSus-100 atlas could play a crucial role in improving the accuracy of DBN segmentation for the research of neurological and psychiatric disease progress and also be helpful for target planning in deep brain stimulation surgery.
\end{abstract}

Keywords: deep brain nuclei, quantitative susceptibility mapping, quantitative susceptibility atlas, multi-modal atlases, basal ganglia, thalamus, parcellation

\title{
1 Introduction
}

Three-dimensional standard space brain atlases are valuable tools as anatomical reference of the human brain (V. Fonov et al., 2011; Horn \& Kühn, 2015; J. Mazziotta et al., 2001). Atlases are widely used to measure neurological disorder related structural changes in patients to compare with the healthy population (Butson, Cooper, Henderson, \& McIntyre, 2007; V.S. Fonov, Evans, McKinstry, Almli, \& Collins, 2009; J.C. Mazziotta et al., 1995; Xiao et al., 2012). Brain atlases that emphasize Deep Brain Nuclei (DBN) structures can 
also be used to refine targets of neurological disease treatment, such as deep brain stimulation (DBS) (Eisenstein et al., 2014; Merkl et al., 2016; Neumann et al., 2015). DBN are closely related to the pathogenesis of neurodegenerative diseases, such as Parkinson's Disease, Dystonia or Obsessive-compulsive Disorder. Faithful identification and depiction of DBN based on neuroimaging is crucial for in-vivo brain structural analysis (Lenglet et al., 2012; Starr et al., 2002; Voges et al., 2002). However, at 3T, conventional T1-weighted (T1w) and T2-weighted (T2w) MR images have difficulties in clearly delineating the bounderies of DBN with respect to surrounding tissues. As well, the image contrast for sub-structures within DBN are also homogeneous. This is mainly because tissue contrast between structures on $\mathrm{T} 1 \mathrm{w} / \mathrm{T} 2 \mathrm{w}$ originates from the differences in signal relaxation rates of the water protons, which are rather weak in DBN. Thus, the existing T1w atlases constructed from healthy population may not ideal for the depiction of DBN of the human brain.

There have been several atlases that were constructed with specific emphasis on the localization of specific brain subcortical nuclei. For example, the DBS intrinsic template atlas (DISTAL atlas) provides subcortical anatomy on the MNI152 nonlinear 2009b templates (Ewert et al., 2018). In this work, three of the most common DBS targets (subthalamic nucleus (STN), internal part of the pallidum (GPi) and ventral intermediate nucleus of the thalamus (VIM)) are localized by co-registering a detailed histological atlas onto the standard T2w template in MNI space. However, the spatial normalization between the individual histology map and the unbiased standard space $\mathrm{T} 2 \mathrm{w}$ template may induce registration deviation. Based on the construction of high-quality T2w unbiased template, the California Institute of Technology (CIT168) atlas provides probabilistic anatomical labels for a bunch of subcortical nuclei that are related to the reward learning and decision making circuit in human brain (Pauli, Nili, \& Tyszka, 2018). In the CerebrA atlas (Manera, Dadar, Fonov, \& Collins, 2019), the authors combined the MNI152 nonlinear 2009b symmetric model (at a resolution of $1 \times 1 \times 1 \mathrm{~mm}^{3}$ ) with the Mindboggle-101 (Klein \& Tourville, 2012), for propagating the labels from Mindboggle-101 to the MNI space through T1w template with an additional manually correction on the subcortical labels. Lau et al. (2020) demonstrated that high resolution (submillimetric) T1 relaxometry measurements at ultra-high field strength (7T) can be used to delineate zona incerta (ZI), a small nucleus in-between STN and Red Nuclei (RN), from surrounding white matter structures. Using this approach, the authors successfully derived in-vivo estimates of the size, shape, location, and tissue characteristics of ZI, confirming observations that was previously only estimated through histological evaluation. Notably, all above mentioned studies benefited from the susceptibility sensitive contrast (T2w or longitudinal T1 relaxometry measurements) that provided better image contrast between DBN and their surrounding tissues.

Recently, a novel post-processing method, referred to as quantitative susceptibility mapping (QSM) (Liu, 2010; Schweser, Deistung, Lehr, \& Reichenbach, 2011; Shmueli et al., 2009; Wharton, Schäfer, \& Bowtell, 2010) has been 
introduced. QSM provides the quantitative measures for iron content of human tissue composition (Bilgic, Pfefferbaum, Rohlfing, Sullivan, \& Adalsteinsson, 2012; Langkammer et al., 2010). QSM is sensitive to the spatial variations of molecular or cellular components that exhibit different magnetic susceptibility properties. Due to its high sensitivity to iron and myelin, QSM provides clear tissue boundaries between iron-rich DBN and myelinated white matter. Thus, QSM enabled the identification of several substructures of the human brain that were in part indiscernible on the other MRI contrasts (i.e. T2w, R2*). As indicated by several recent studies (Haacke, Tang, Neelavalli, \& Cheng, 2010; Hanspach et al., 2017; Schweser et al., 2011; Shmueli et al., 2009), common DBS targets, including substantia nigra pars reticulata ( $\mathrm{SNr})$, VIM, STN and the substructures of the GP, were clearly distinguishable from surrounding tissues on QSM. Thalamus also extracted plenty of attentions in QSM related studies, such as in Schweser et al. (2018) and Hanspach et al. (2017), both authors proposed to depict sub-nuclei in thalamus directly based on the contrast of group-wisely averaged QSM template. Deistung et al. (2013) indicated that using high-resolution QSM image acquired at 7T, QSM exhibited superb contrast that allowed histological-consistent identification for substructures of the thalamus, midbrain and basal ganglia.

There are a few susceptibility atlases proposed for facilitating DBN parcellation (X. Li et al., 2019; Lim et al., 2013; Zhang et al., 2018). Lim et al. (2013) added a QSM template to the single-subject Eve atlas space, to extend Eve atlas with accurate "deep gray matter parcellation map". Limited by low image Signal to Noise Ratio (SNR) of the single image QSM atlas, the DBN regions were annotated as a whole part in Eve QSM atlas. Zhang et al. (2018) proposed a longitudinal age-dependent QSM atlas, providing an efficient tool for segmenting brain subcortical structures from individual QSM images for variety of age-range. However, restricted by the image resolution $\left(1 \times 1 \times 1 \mathrm{~mm}^{3}\right)$ of the 3T QSM template, thalamus was parcelled into only five sub-regions in each hemisphere. X. Li et al. (2019) proposed a multi-atlas strategy for achieving a more reliable automated tissue susceptibility quantification framework in series of typical DBN. However, to the best of our knowledge, an unbiased MNI-space version and a histological-consistent DBN sub-region parcellation map were not available in these QSM templates.

For standard MNI-space, the most widely used atlases are Colin-27 atlases (Holmes et al., 1998) and ICBM-152 (Ewert et al., 2018) atlases. Colin-27 includes T1w/T2w atlases with a normal-resolution version $\left(1 \times 1 \times 1 \mathrm{~mm}^{3}\right)$ and a high-resolution version $\left(0.5 \times 0.5 \times 0.5 \mathrm{~mm}^{3}\right)$. In the MNI152 nonlinear $2009 \mathrm{~b}$ version, $\mathrm{T} 1 \mathrm{w} / \mathrm{T} 2 \mathrm{w} / \mathrm{PD}$ and T2-relaxometry atlases are available. Specially, the two groups of atlases are both constructed from normative young adult population: Colin-27 (a 28-years individual) (Holmes et al., 1998) and ICBM152 (18.5-43.5 years individuals) (Ewert et al., 2018). This is partially because the MR image quality is sensitive to motion artifact during image scanning. In order to minimize the effect of motion on image quality and thus further 
facilitate the atlas construction process, normative young adult subjects are adopted in adult brain atlas construction research works.

Towards this end, we propose to construct an unbiased QSM atlas in MNI space, using multi-modal MR images (including T1w, Gradient Echo (GRE) magnitude and QSM) collected from 100 young healthy individuals (age 19-29 years). In our strategy, T1w image provides clear GM-WM contrast and acts as the guidance towards MNI space; GRE magnitude is used for normalizing T1w and QSM spatial information; while QSM performs superb contrast for inside and outside DBN and highlights positions for iron-rich nuclei, and especially guides detailed DBN sub-region identifications in the MNI space. The averaging process for spatially normalizing individual MR images substantially enhanced the image SNR in the unbiased QSM template. We further apply border-enhancement technique to emphasize the boundaries of DBN and then propose a series of DBN delineation, including thirty-two sub-thalamic nuclei on each hemisphere of thalamus (Thal), and another eleven-pairs of bilateral basal ganglia nuclei: putamen $(\mathrm{Pu})$, nucleus accumbens (NAcc), caudate nucleus (Ca), ventral pallidum (VP), internal and external globus pallidus (GPi \& GPe), Red Nucleus (RN), pars reticulata and pars compacta of substantia nigra (SNr \& SNc) and subthalamic nucleus (STN). Specially, thalamus depiction is conducted on a handcrafted parcellation map on an individual 7T QSM image following the Schaltenbrand and Wahren histology atlas, then carefully warped into $3 \mathrm{~T}$ individual space and weighted fused into MNI space. To our best knowledge, the detailed histological-consistent thalamic nucleus parcellation map is defined in MNI space for the first time. For assessing the feasibility of the MuSus-100 atlas, we additionally segment 20 individual subjects using the proposed atlas and then calculate quantitative parameters by comparing segmentation result with ground truth manual annotations. The result show that the MuSus-100 atlas outperforms existing atlases on atlasguided individual brain image DBN segmentation accuracy and robustness. We also apply the proposed atlas on the result of voxel-wise PD QSM image analysis, to identify the pathology-related iron content difference in small-size DBN sub-regions between PD patients and healthy control subjects. Abnormalities in iron content are found in the bilateral $\mathrm{Ca}, \mathrm{Pu}, \mathrm{GP}$,Thal, RN and left STN and SN (both SNr and SNc). In thalamus, the ventral and the central sub-regions of show the most essential PD-related brain tissue susceptibility variation.

\section{Methods}

This section describes the detailed processes of the MNI-space unbiased QSM atlas construction and the parcellation of DBN, mainly including the steps of participant recruitment, image acquisition, atlas construction, DBN parcellation, evaluation criterion and application of proposed atlas. 


\subsection{MR Image acquisition and reconstruction}

Data for atlas construction. One hundred healthy volunteers (41 males, 59 females) were recruited for MR data collection. The mean age of the volunteers is 24.03 (range from 19 to 29 years old). The data were acquired on a 3T GE750 MRI scanner. Each individual underwent the scanning sessions of T1-weighted (T1w) sequence and a multi-echo, spoiled-gradient recalled (SPGR) sequence. The scanning parameters of the $\mathrm{T} 1 \mathrm{w}$ image: repetition time $(\mathrm{TR})=7.336 \mathrm{~ms}$; echo time $(\mathrm{TE})=3.036 \mathrm{~ms}$; flip angle $=11^{\circ}$; field of view $(\mathrm{FOV})=260 \times 260 \mathrm{~mm}^{2}$; matrix size $=256 \times 256$; slice thickness $=1.2 \mathrm{~mm} ; 196$ continuous sagittal slices. A three-dimensional SPGR sequence was utilized to obtain QSM images with the following parameters: $\mathrm{TR}=33.7 \mathrm{~ms}$, TE1/spacing $/ \mathrm{TE} 8=4.56 / 3.65 / 30.11$ $\mathrm{ms}$, flip angle $=20^{\circ}, \mathrm{FOV}=240 \times 240 \mathrm{~mm}^{2}$, matrix size $=416 \times 384$, slice thickness $=2 \mathrm{~mm}$, resolution $=0.47 \times 0.47 \times 2 \mathrm{~mm}^{3}$. All the images were resampled to the same resolution of $1 \times 1 \times 1 \mathrm{~mm}^{3}$ through the operations in $\mathrm{k}$-space. The raw phase was unwrapped using the Laplacian-based phase unwrapping, and the normalized background phase was removed by V-SHARP (Wu, Li, Guidon, \& Liu, 2012)). The susceptibility maps were determined by the STAR-QSM algorithm (Wei et al., 2015). All scans are inspected to screen out the images with incomplete brain structure or with obvious motion artifact.

Data for thalamus sub-nuclei delineation. A high-resolution QSM is scanned on a 7T GE950 MRI scanner using a multi-echo GRE sequence (TE1/spacing/TE4=5/7.8/28.4 ms, TR=34 ms, FA=8, $\mathrm{BW}=160 \mathrm{~Hz} / \mathrm{px}$, matrix $=576 \times 414 \times 256$, resolution $\left.0.4 \times 0.4 \times 0.4 \mathrm{~mm}^{3}\right)$. QSM reconstruction was performed using the same technique as described above.

Data for atlas-guided individual $D B N$ segmentation accuracy evaluation. 20 individual subjects with variable age (25-65 years old, 9 female, 11 male) were scanned on a 3T GE750 MRI scanner for T1w and QSM. T1w images were acquired using the following parameters: $\mathrm{TR}=2300 \mathrm{~ms}$; $\mathrm{TE}=1.95$ ms; flip angle $=9^{\circ} ; \mathrm{FOV}=256 \times 256 \mathrm{~mm}^{2}$; matrix size $=256 \times 256$; slice thickness $=1 \mathrm{~mm} ; 152$ continuous sagittal slices. QSM were performed using 3D multi-echo GRE sequence: TR/TE1/TE spacing=41.6/3.2/2.2 ms; number of echoes $=16$; flip angle $=12^{\circ} ; \mathrm{FOV}=256 \times 256 \mathrm{~mm}^{2} ;$ matrix size $=256 \times 256$; slice thickness $=1 \mathrm{~mm}$. The manually DBN annotations in the individual QSM images by two radiologists serves as ground truth (GT) for individual image segmentation accuracy evaluation.

Data for PD-related brain iron content abnormality analysis. QSM scans of 87 subjects with $47 \mathrm{PD}$ patients $(56.9 \pm 5.0$ years old, 26 female, 21 male) and 40 health controls (57.4 \pm 6.3 years old, 18 female, 22 male) were conducted on 3T GE750 MRI scanner. We used threedimensional SPGR sequence with the following parameters: $\mathrm{TR}=33.7 \mathrm{~ms}$, TE1 $/$ spacing $/ \mathrm{TE} 8=4.56 / 3.65 / 30.11 \mathrm{~ms}$, flip angle $=20^{\circ}, \mathrm{FOV}=240 \times 240 \mathrm{~mm}^{2}$, matrix size $=416 \times 384$, slice thickness $=2 \mathrm{~mm}$, resolution $=0.47 \times 0.47 \times 2 \mathrm{~mm}^{3}$.

All procedures of the present study were in accordance with the Declaration of Helsinki and were approved by the Ethics Committee of the Second Affiliated 
Hospital, Zhejiang University School of Medicine. The participants were all informed of the study procedures and gave their written consent.

\subsection{Atlas construction}

The atlas construction includes four steps: pairwise image registration, hybrid image generation, native space atlas construction, and MNI space projection. The pipeline is illustrated in the Figure 1.

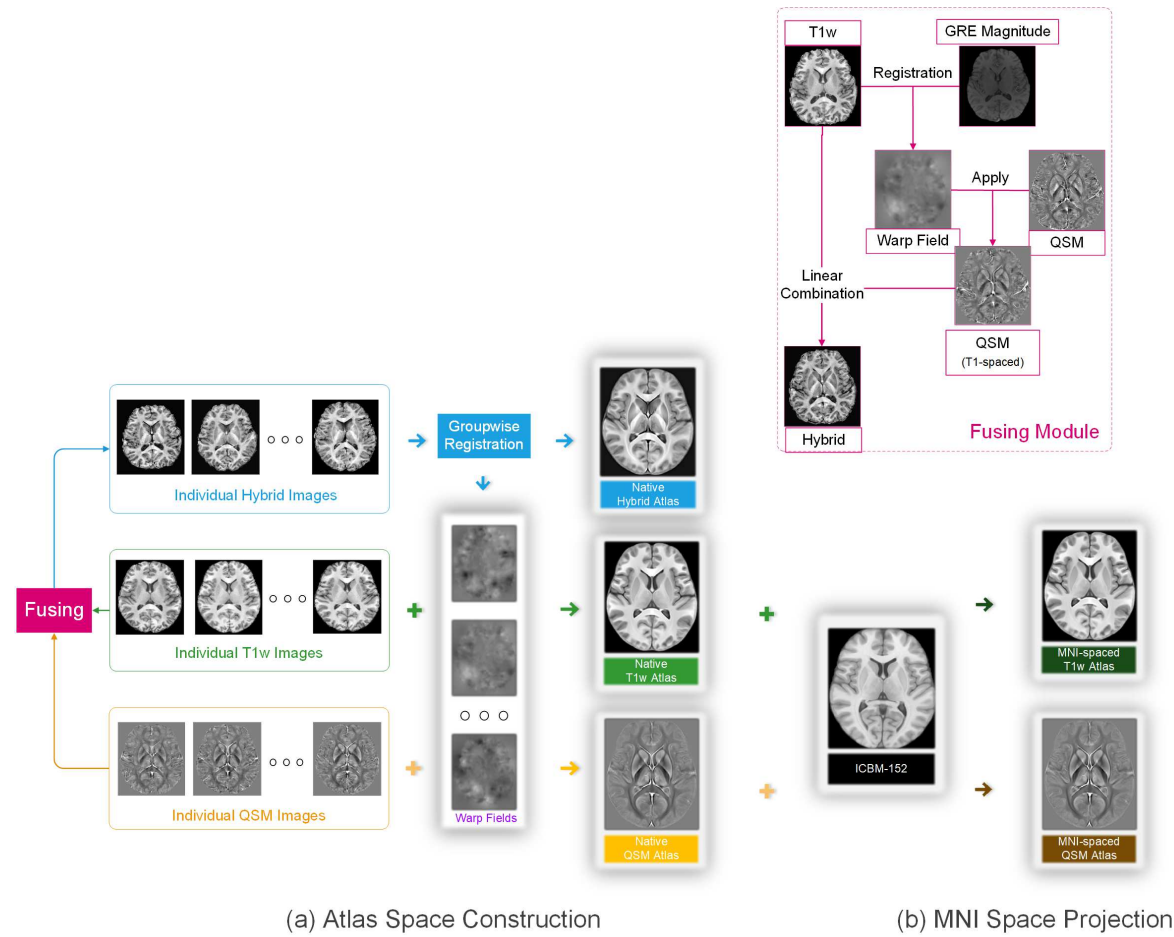

Fig. 1 Pipeline of MNI space unbiased QSM atlas construction. (a) Atlas construction in native space. Individual hybrid images are fused from individual T1w and QSM images. The fusing module is shown in the right-top box, indicating the pairwise image registration between individual T1w and QSM images for generating the hybrid image. Group-wise registration is then applied on individual hybrid images to generate hybrid atlas image and warp field for each subject. (b) Atlas projection into MNI space. Using T1w atlas in native space and MNI space as intermediate, the QSM atlas is projected into the MNI space.

\subsubsection{Pairwise image registration}

Pairwise image registration aligns the moving image $\left(I^{M}\right)$ to the fixed image $\left(I^{F}\right)$. The registration process includes similarity optimization and application of transformation, which can be mathematically expressed as: 
$\phi^{M \rightarrow F}=\arg \min _{\phi} \mathcal{L}_{\operatorname{sim}}\left(I^{F}, \phi^{M \rightarrow F} \circ I^{M}\right)+\lambda \mathcal{L}_{\text {smooth }}\left(\phi^{M \rightarrow F}\right)=f_{\operatorname{sim}}\left(I^{F}, I^{M}\right)$

where $\mathcal{L}_{\text {sim }}$ and $\mathcal{L}_{\text {smooth }}$ denotes the similarity metric to minimize and the regularization term to keep transformation $\phi$ smooth. The output $\phi$ denotes the compound transformation of linear and nonlinear transformations. Combining the optimizing process and the warping process, we simply mark the registration with moving image $I^{M}$ to fixed image $I^{F}$ as

$$
I^{M \rightarrow F}=\phi^{M \rightarrow F} \circ I^{M}=f_{\mathrm{reg}}\left(I^{F}, I^{M}\right)
$$

\subsubsection{Native space T1w and QSM image pairwise registration}

Each individual has MR images of T1w, QSM, and GRE magnitude (Mag), where QSM and GRE magnitude are both acquired based on multi-echo GRE sequence thus share the same coordinate space. For $\mathrm{T} 1 \mathrm{w}$ image, the bias field correction is conducted using N4 intensity normalization (Tustison et al., 2010), and the skull removal is performed using Brain Extraction Tool (BET) (Smith, 2002). For each individual $i,(i \in[1, N]$ denotes the $i$-th individual, $N$ denotes the total number of subject), the GRE magnitude map $I_{i}^{\mathrm{Mag}}$ is registered to the $\mathrm{T} 1 \mathrm{w}$ image $I_{i}^{\mathrm{T} 1 \mathrm{w}}$ using pairwise image registration. Then the transformation was applied to the QSM image $I_{i}^{\mathrm{QSM}}$ to warp it to the coordinate space of $\mathrm{T} 1 \mathrm{w}$ image, expressed as $I_{i}^{\mathrm{QSM} \rightarrow \mathrm{T} 1 \mathrm{w}}=\phi_{i}^{\mathrm{Mag} \rightarrow \mathrm{T} 1 \mathrm{w}} \circ I_{i}^{\mathrm{QSM}}$. The operation of image alignment can be summarized as

$$
I_{i}^{\mathrm{QSM} \rightarrow \mathrm{T} 1 \mathrm{w}}=f_{\mathrm{align}}\left(I_{i}^{\mathrm{QSM}} \mid I_{i}^{\mathrm{T} 1 \mathrm{w}}, I_{i}^{\mathrm{Mag}}\right)
$$

\subsubsection{Hybrid image generation}

After pairwise registration, the hybrid images are generated by fusing $\mathrm{T} 1 \mathrm{w}$ and QSM images to enhance the DBN representation in the T1w images, and also to promote the cortical WM/GM (white matter/gray matter) contrast in QSM. For each individual, the hybrid image $I_{i}^{\text {hybrid }}$ is synthesized via a fusion process $f_{\text {fusion }}(\cdot)$, which is a linear combination of the aligned image pairs $\left(I_{i}^{\mathrm{T} 1 \mathrm{w}}, I_{i}^{\mathrm{QSM}} \rightarrow \mathrm{T} 1 \mathrm{w}\right)$, expressed as

$$
I_{i}^{\mathrm{hybrid}}=f_{\text {fusion }}\left(I_{i}^{\mathrm{T} 1 \mathrm{w}}, I_{i}^{\mathrm{QSM} \rightarrow \mathrm{T} 1 \mathrm{w}}\right)=I_{i}^{\mathrm{T} 1 \mathrm{w}}+\alpha I_{i}^{\mathrm{QSM} \rightarrow \mathrm{T} 1 \mathrm{w}}
$$

where $\alpha$ is a scalar controlling the fusion extend. The larger the $\alpha$ is, the stronger the visual contrast of DBN would be in the fused image since the QSM image weights more in the hybrid image. After multiple trials, $\alpha=12500$ is empirically selected by visual inspection, using which the DBN regions in hybrid image show the sharpest contrast. 


\subsubsection{Native space atlas construction}

Unbiased group-wise registration is performed using antsMultivariateTemplateConstruction2 utility implemented in Advanced Normalization Tools (ANTs) registration toolkit (Avants, Tustison, \& Song, 2009). The initial template $T^{(t=1)}$ (where $(t=m)$ denotes the $m$-th iteration count) is defined as the original individual image that is the most similar to all the other images in the dataset for atlas construction. Then each original individual image $I_{i}^{(t=0)}$ is updated to the current template $T^{(t=m)}$ at the $m$-th iteration by registering to the template as

$$
I_{i}^{(t=m)}=f_{\mathrm{reg}}\left(T^{(t=m)}, I_{i}^{(t=0)}\right) .
$$

Finally, the template is updated by voxel-wise averaging of each warped individual image $I_{i}^{(t=m)}$ as

$$
\bar{T}^{(t=m+1)}=\frac{1}{N} \sum_{i=0}^{N} I_{i}^{(t=m)}
$$

Notice that in practice, further processing such as image sharpening is included in the averaging process. Generally, we mark the averaging process as $\bar{T}=$ $\operatorname{avg}\left(\boldsymbol{\Phi} \circ \mathbf{I}^{(t=0)}\right)$, where $\boldsymbol{\Phi}=\left\{\phi_{i} \mid i=1,2, \cdots, N\right\}$ and $\mathbf{I}=\left\{I_{i} \mid i=1,2, \cdots, N\right\}$, respectively denotes the set of deformation fields from individual to atlas space and the set of individual images.

Update of the average template to achieve performance of sharpness in atlas construction Additional post-processing steps are applied to the average atlas to achieve a refined template update. We mark the process as $T^{(t=m+1)}=\operatorname{update}\left(\bar{T}^{(t=m+1)}, \boldsymbol{\Phi}^{-\mathbf{1}}\right)$, where $T$ denotes the average template at any interation $t=m$ and $\boldsymbol{\Phi}^{-\mathbf{1}}$ represents the inverted warp field set at the same iteration. The detailed process includes:

1. Voxel-wise averaging of the nonlinear inverse warp fields $\mathbf{\Phi}^{-\mathbf{1}}$, which can be considering as the degree of brain structure variation among population used for atlas construction.

2. Multiplying a factor that control the deformation degree to the averaged inverse warp fields.

3. Applying transformations to the averaged atlas, including applying the inverse linear transformations once and applying the average inverse warp field.

Atlas creation pipeline In each iteration, the full process of native space QSM atlas construction, including hybrid image generation, group-wise registration and average template update, is summarized in the Algorithm 1 below.

Multiple modality atlas generation Along with the construction of the hybrid template, the transformations $\boldsymbol{\Phi}=\left\{\phi_{i}\right\}$ from the individual space to the final atlas space are also saved. Then the T1w and QSM atlases are created by leveraging the transformations of the hybrid atlas to the corresponding 


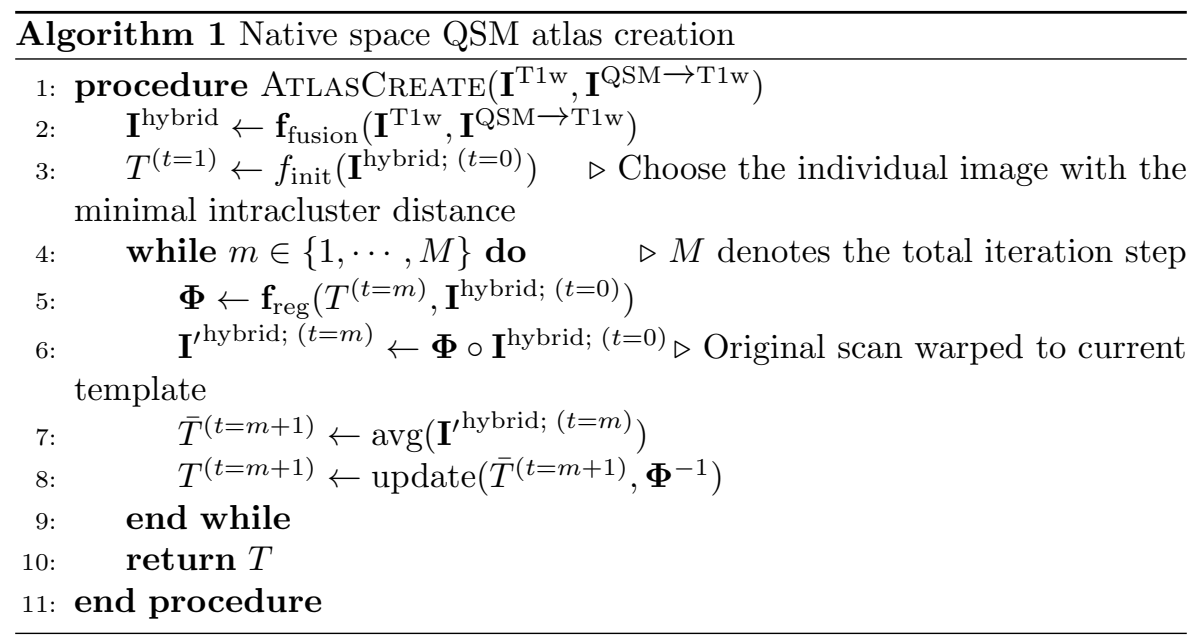

individual images and then averaging the warped individual scans, which is denoted as:

$$
\begin{gathered}
T^{\mathrm{T} 1 \mathrm{w}}=\operatorname{avg}\left(\boldsymbol{\Phi} \circ \mathbf{I}^{\mathrm{T} 1 \mathrm{w}}\right) \\
T^{\mathrm{QSM}}=\operatorname{avg}\left(\boldsymbol{\Phi} \circ \mathbf{I}^{\mathrm{QSM} \rightarrow \mathrm{T} 1 \mathrm{w}}\right)
\end{gathered}
$$

Notice that only the averaging process is performed while the update process in atlas construction is not performed, since updating is an plug-in process during the atlas creation process.

\subsubsection{Towards MNI space projection}

For projecting our unbiased QSM atlas into the standard MNI space, we firstly performed pairwise registration between our $\mathrm{T} 1 \mathrm{w}$ atlas and the modalityidentical standard MNI-spaced T1w atlas (ICBM-152 nonlinear 2009c, resolution of $1 \times 1 \times 1 \mathrm{~mm}^{3}$ ) (Ewert et al., 2018). For ICBM-152 T1w atlas, skull is removed using the official brain tissue mask. Then we warp our T1w atlas towards the ICBM-152 brain atlas via non-linear registration. In this process, the deformation field is saved and then applied to the native space unbiased QSM atlas.

\subsection{Parcellation creation}

\subsubsection{DBN border-enhancement and manual parcellation}

To further improve the accuracy and the objectivity for our atlas, we apply techniques of border-enhancement to assist the depiction. The borderenhancement is carried by applying $3 \mathrm{D}$ volumetric Laplacian kernel to the constructed QSM atlas. The implemented Laplacian kernel is defined as:

$$
\left[\begin{array}{ccc}
0 & 0 & 0 \\
0 & -1 & 0 \\
0 & 0 & 0
\end{array}\right],\left[\begin{array}{ccc}
0 & -1 & 0 \\
-1 & 6 & -1 \\
0 & -1 & 0
\end{array}\right],\left[\begin{array}{ccc}
0 & 0 & 0 \\
0 & -1 & 0 \\
0 & 0 & 0
\end{array}\right]
$$


The above kernel is convoluted with both QSM and T1w template to yield QSM and T1w Laplacian map. For DBN annotation, the generated templates and Laplacian maps are all used as reference, where the QSM template and Laplacian map are the main reference due to the clear contrast in iron-rich DBN.

Two experienced radiologists have been involved in the delineating work, for eliminating subjectivity from single-rater delineating. Considering that a common initialization often leads more consistent manual delineation between different raters, a full-brain parcellation map AAL3 (Rolls, Huang, Lin, Feng, \& Joliot, 2020) that contains DBN region annotations is firstly overlaped on the MNI-spaced unbiased QSM template. AAL3 contains less DBN regions than that we propose, the rest ROIs are defined based on QSM template contrast. Then, the delineating work is carried by the two raters in isolation, where communications only take place after the delineation work. The final DBN annotations for the proposed atlas are carried out by applying the consensus of the delineated parcellation maps from the two raters, regarding the objections are solved during the after-work communication which anatomical consensus has reached and the differences only represents occasional delineation mainly on the boundaries. Specially, a voxel is assigned to a region only if both raters labeled the voxel as the same region.

The labeled nuclei include the bilateral Pu, Ca, GPi, GPe, RN, SNr, SNc, NAcc, STN and Thal. For the nuclei located adjacent to the telencephalon, such as GP (GPi and GPe), Pu and Ca, the main reference is T1w atlas, while QSM atlas is used for auxiliary outline positioning. The $\mathrm{Ca}$ and NAcc are mainly delineated in the sagittal view with respect to the division of the lower boundary of CSF. The lower fiber bundle is considered in $\mathrm{Pu}$ parcellation. The Ca, GPi and GPe were mainly labeled in the axial view and are closely attached. The QSM atlas is mainly referenced in the labeling work of iron-rich DBN, including the STN, RN, and SN (SNr and SNc). The parcellation of STN is mainly defined in the axial view with the spindle shape. The parcellation of spatially close SN is defined with respect to the STN, following the guidance of previous research and histological atlases (Behrens et al., 2003; He et al., 2017; Morel, Magnin, \& Jeanmonod, 1997).

\subsubsection{Construction of thalamic subnuclei annotations based on high-resolution QSM image and label fusion method}

The process of thalamic subnuclei parcellation is shown in Figure 2. Firstly, a high-resolution (h-reso) QSM image $\left(0.4 \times 0.4 \times 0.4 \mathrm{~mm}^{3}\right)$ is scanned using a $7 \mathrm{~T}$ scanner using parameter presented in section 2.1 . Then a detailed thalamic subregion parcellation map is created by the two radiologists referring to Schaltenbrand and Wahren histology atlas, with assist of the border-enhanced technique. The h-reso QSM image is then aligned to the 100 low-resolution (1reso) QSM image dataset for compensation the single individual image bias. For registration detail, l-reso images are firstly up-sampled to the size of hreso image in order to reduce the influence of resolution difference between 
scans, and then they are registered to the h-iso image using non-linear registration (SyN) (Avants, Epstein, Grossman, \& Gee, 2008). Then the thalamic sub-nucleus parcellation map is correspondingly propagated into each l-reso individual space via applying the inverted deformation fields and downsampled to the size of l-reso individual images. Thus, for each individual l-reso QSM image, a detailed thalamic parcellation map is created. Then, as shown in Figure 2, based on the deformation fields from QSM atlas construction, joint label fusion (Wang et al., 2012) is performed on each individual thalamic parcellation map. Finally, the 100 individual thalamic sub-nucleus parcellation maps were fused to form a 64-ROI parcellation of bilateral thalamus in $3 \mathrm{~T}$ atlas space.

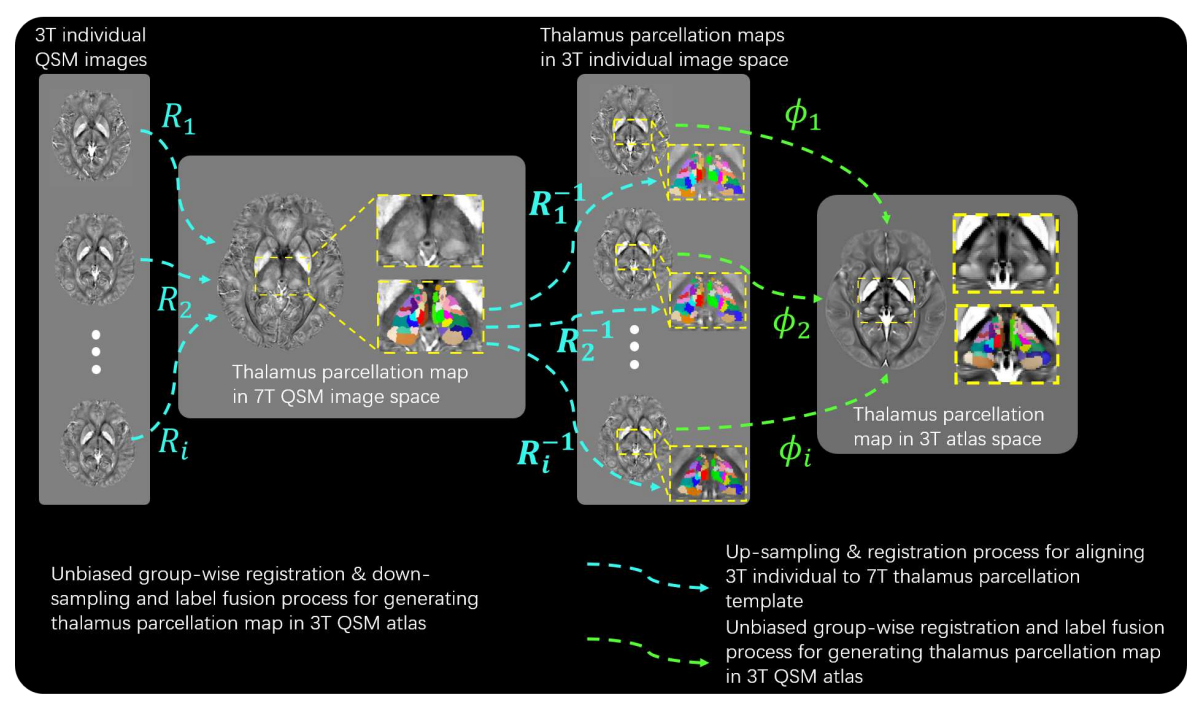

Fig. 2 Pipeline for creating the thalamic subnuclei in atlas space. The lowresolution individual QSM images are warped to the high-resolution QSM image space, and then the parcellation map is inversely warped to each individual's image, in order to form thalamic parcellation. Finally, the detailed thalamus parcellation map for atlas space is generated by joint fusion.

\subsection{Evaluations on atlas construction quality and DBN parcellation map accuracy}

\subsubsection{Comparison of image contrast inside and outside DBN across different atlases}

High image contrast between brain DBN sub-regions and the surrounding brain tissues facilitates delineation for DBN sub-region boundaries. We extracted intensity profile lines across selected image patches that containing DBN regions to compare DBN image contrast of atlases built from different strategies. More intensive variation along the profile line suggests higher contrast 
in image intensity. In addition, we conduct k-means intensity clustering on selected atlas slices to generate rough local parcellation maps that work as an initialization for DBN delineation. More accurate and detailed clustering result suggests more clear contrast between DBN regions.

\subsubsection{Analysis of using parcellation map to delineate DBN in individual images}

In practice, manual parcellation is usually needed for individual brain image DBN delineation, especially for small-size nuclei. Wrapping the predefined parcellation map to the individual images is a more efficient way of DBN delineation. The process includes registering the individual images to the atlas and then applying the inverse warping of the atlas-space parcellation map back to the individual images. We assumed that the DBN parcellation of our atlases would be more accurate than those of the previous atlases.

For evaluation, we computed the Dice coefficient (DSC), Kappa coefficient, and Hausdorff distance between the inverse warping of the atlas-spaced parcellation map and the manual delineation of DBN in 20 individuals.

The Dice coefficient measures the overlap proportion between the warped parcellation and the GT. DSC ranges from 0 to 1 . A Dice coefficient closer to 1 indicates that the warped parcellation overlaps more with the GT. The Dice coefficient between parcellation $A$ and $B$ is defined as

$$
D S C(A, B)=\frac{|A \cap B|}{|A+B|}
$$

The Hausdorff distance measures the maximal element distance between the warped parcellation and the GT. The Hausdorff distance between parcellation $A$ and $B$ is defined as

$$
H D(A, B)=\max _{a \in A} \min _{b \in B} a-b_{2}
$$

$\mathrm{Ca}, \mathrm{Pu}, \mathrm{GPe}, \mathrm{GPi}, \mathrm{NAcc}, \mathrm{SNc}, \mathrm{SNr}, \mathrm{RN}, \mathrm{VP}$ and STN are the nuclei of interest. The following atlases and parcellations are selected for comparison with ours: Colin-27 atlas (Holmes et al., 1998) with AAL3 parcellation (version 3.1, updated in June 2020) (Rolls et al., 2020), Zhang's longitudinal QSM atlas with its parcellation (Zhang et al., 2018), ICBM-152 2009c asymmetric T1w atlas (V. Fonov et al., 2011) with CIT-168 subcortical reinforcement learning parcellation (Pauli et al., 2018), AHEAD ICBM-aligned QSM template with its atlas (Alkemade et al., 2020). Based on the different nuclei included and the different imaging properties of the atlases and parcellations, we categorized them into three groups: the coarse DBN parcellation group, the fine DBN parcellation group, and the high-resolution DBN parcellation group.

The compared atlases and parcellations of the coarse-parcellation group include Colin-27 with AAL3 and Zhang's atlas with parcellation. The Colin-27 T1w and Zhang's QSM atlases are both acquired in 3T scanners with a voxel resolution of $1 \times 1 \times 1 \mathrm{~mm}^{3}$. Colin-27 is in the MNI-space, and Zhang's atlas is 
in native atlas space. In this group, the nuclei of Cau, Pu, GP, NAcc and SN are involved for comparison.

The fine-parcellation group includes the ICBM-152 2009c Asymmetric atlas and CIT168 parcellation in comparison. The parcellation of CIT168 is a probability map, and thus we sampled it to binary map with a threshold of 0.5 , as the original publication suggests. The left- and right-hemisphere nuclei are differently marked, for the depiction of the left- and right-hemisphere nuclei are not separated in the original parcellation. The comparison of this group is still intra-modality, respectively, with our QSM template and ICBM 2009c T1w atlas.

The high-resolution group only includes the AHEAD atlas only in comparison. The AHEAD atlas is created on multi-modal 7T individual QSM scans with the spacing of $0.5 \times 0.5 \times 0.5 \mathrm{~mm}^{3}$.

\subsection{Application: revealing the PD-related pathology abnormality of iron content in DBN}

In this section, we apply the novel proposed QSM atlas and DBN parcellations to identify the PD-related iron content abnormality specially in deep brain regions. Firstly, we perform a whole-brain voxel-wise statistical comparison of susceptibility value between PD patients and normal controls (NC). All individual QSM scans are warped to the MNI space by co-registration to the proposed QSM atlas. A smoothing operation is conducted by applying a 3D Gaussian kernel with a standard derivation of $3 \mathrm{~mm}$ (Betts, AcostaCabronero, Cardenas-Blanco, Nestor, \& Düzel, 2016), in order to further prevent the potential registration error. Then the statistical examination is performed by a two-sample t-test using the randomise utility implemented in FSL (Winkler, Ridgway, Webster, Smith, \& Nichols, 2014; Woolrich et al., 2009) with 5,000 permutations and threshold-free cluster enhancement. The significance threshold is set at $P<0.05$ with family-wise error (FWE) corrected. Before the statistical examination, the covariates of age and gender are firstly tested whether to be irrelevant across the groups by examining the null hypothesis that the mean values of the tested covariates show no significant differences between groups. Then, the two groups of scans are tested with the null-hypothesis of voxel-wise group medians of susceptibility value are equal. If covariates show a significant difference between the groups, i.e., the null hypothesis regarding covariates cannot be rejected, the covariates are added as additional variables for a covariate-adjusted test. Our parcellation map is applied to identify the PD-related nuclei and sub-nuclei that shows significance value in permutation test.

\section{Results}

This section firstly illustrates the atlas construction performance conducted based on the hybridization strategy. Then the comparison of image contrast among the atlases is presented. Thirdly, it shows the manual segmentation of 
the DBN and the thalamic subnuclei on the atlases. The evaluation of individual image $\mathrm{DBN}$ parcellation accuracy and application in $\mathrm{PD}$ iron content analysis are presented in the last part.

\subsection{Atlas construction}

The atlases constructed from the hybrid, T1w and QSM images are shown in Figure 3, together with the T1w atlas and the QSM atlas that built without image hybridization guidance. As illustrated, the $\mathrm{T} 1 \mathrm{w}$ atlas shows a clear contrast of gyri and sulci, and the QSM atlas shows clear boundaries of the iron-rich DBN. The T1w-QSM hybrid atlas combines the advantages of the T1w and the QSM atlases and shows high image contrast in both the cortices and the DBN. Compared with the T1w atlas, the depiction of the DBN, such as the thalamus and $\mathrm{SN}$, is greatly improved in the hybrid atlas.
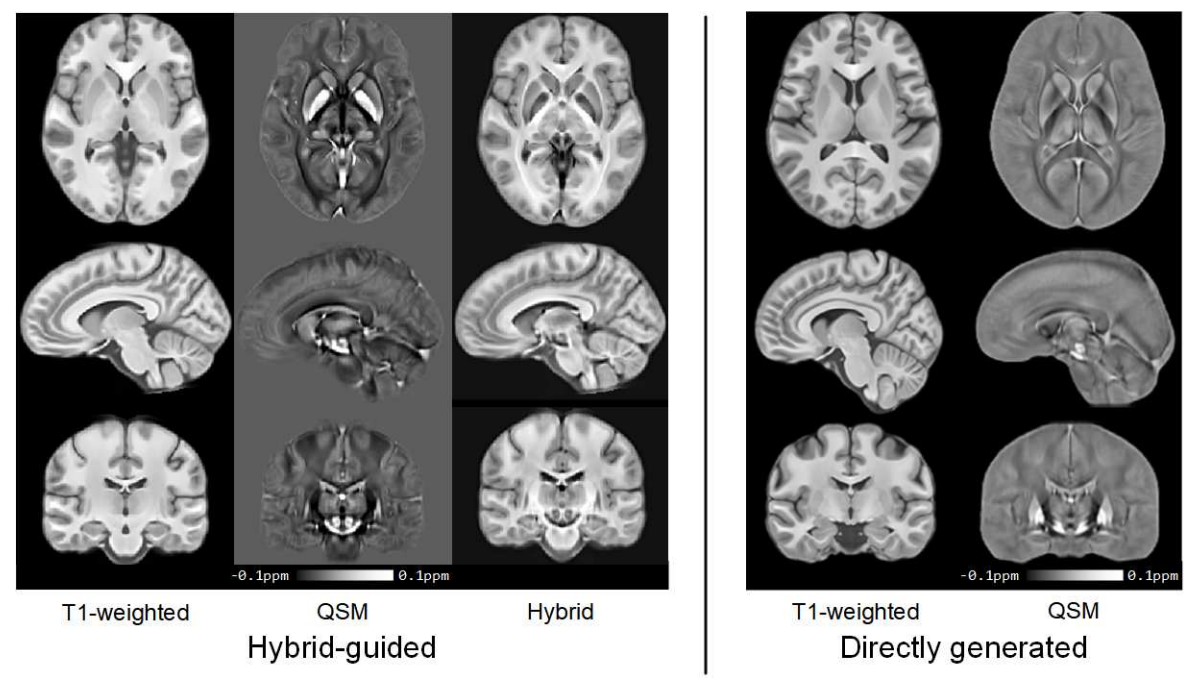

Fig. 3 Qualitative comparison of the hybrid-guided (ours approach) atlases and the directly constructed atlases. All atlases are presented in the MNI-152 coordinate space. Range of QSM scan is from -0.1ppm to 0.1ppm.

\subsection{Contrast analysis of multi-modal hybrid atlas}

We compared the intensity contrast among the constructed atlases. The indices for contrast evaluation in the DBN regions include the result of the borderenhanced display with profile line analysis, and the k-means intensity clustering based segmentation. The T1w, QSM, multi-modal hybrid-guided atlases, and the ICBM-152 T1w atlas were taken for comparison. Two patches containing the DBN of interest in the basal ganglia and thalamus were used for the borderenhanced display and the profile line analysis. 
The border-enhanced display and the profile line results are shown in Figure 4 and Figure 5 respectively. The profile lines are plotted along the intensity scan of a specific row crossing different nuclei on the border-enhanced figures. A large fluctuation in intensity indicates high contrast between the nuclei and the surrounding brain tissues.

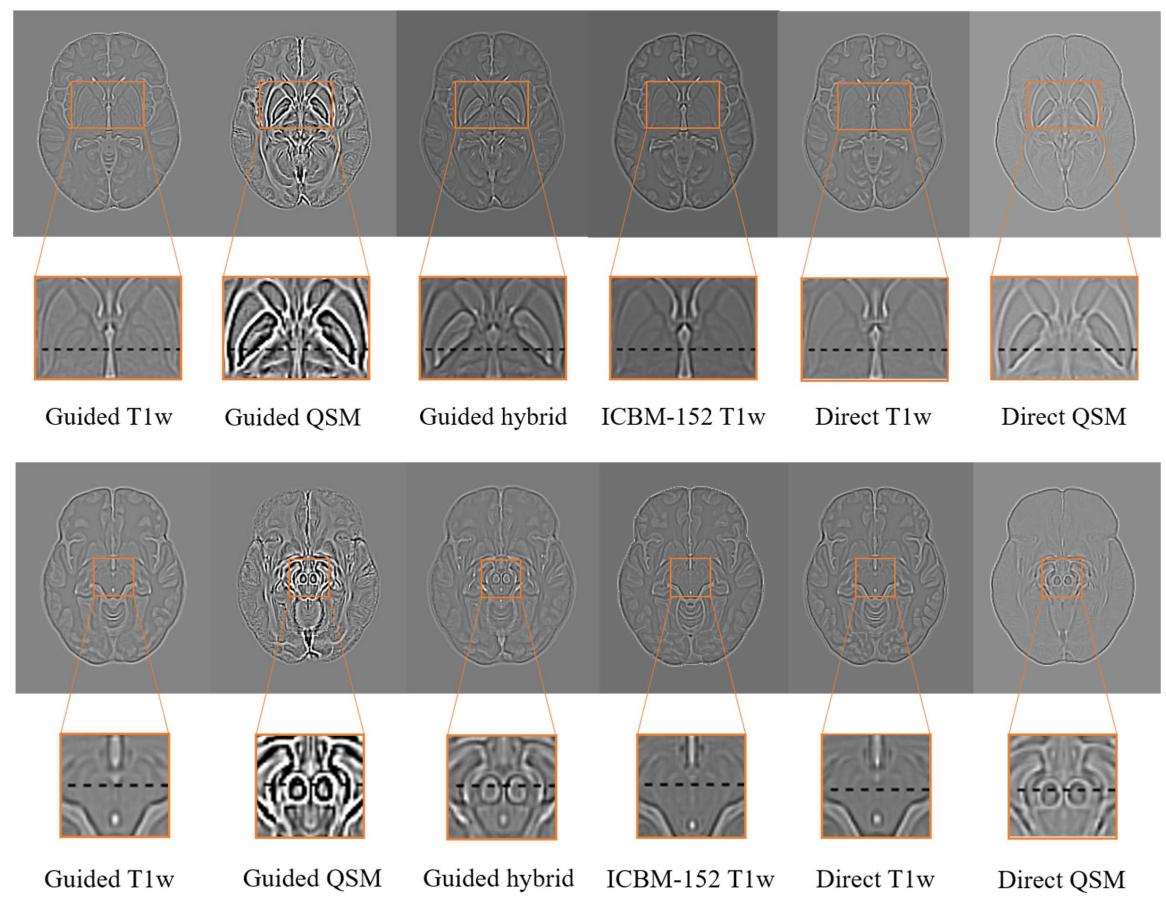

Fig. 4 Border-enhanced displays of atlases at a selected slice using the Laplacian kernel. Some structures in the basal ganglia $(\mathrm{Ca}, \mathrm{Pu}$, and GP) and the Thal are included in the slice of the upper panel. Some structures in the basal ganglia ( $\mathrm{SN}$ and $\mathrm{RN}$ ) are included in the slice of the lower panel. The areas within the orange rectangular are zoomed in for clear views. The image intensity distributed along profile lines that go through the enlarged view of ROI patches are shown in Figure 5.

As shown in Figure 4, both T1w atlases (ICBM-152 and ours guided $\mathrm{T} 1 \mathrm{w})$ provide clear boundaries of the nuclei of $\mathrm{Ca}, \mathrm{Pu}$, and $\mathrm{GP}$, as well as a coarse border of Thal. The division between GPe and GPi can also be observed on both T1w atlases. Our QSM atlas depicts the nuclei that are hard to be observed in T1w atlases, such as the RN and SN. The contrast of borders of other DBN, such as $\mathrm{Ca}, \mathrm{Pu}$, and GP (external \& internal), is also enhanced. The boundary of the thalamic subnuclei is also more clearly observed on the QSM atlas than that in the T1w atlases. However, the QSM atlas displays poorer contrast at cortical regions than the $\mathrm{T} 1 \mathrm{w}$ atlases. The hybrid atlas combines the advantages of the T1w and QSM atlases, showing sharp contrast in both cortical and subcortical regions. The cortical features in the hybrid 

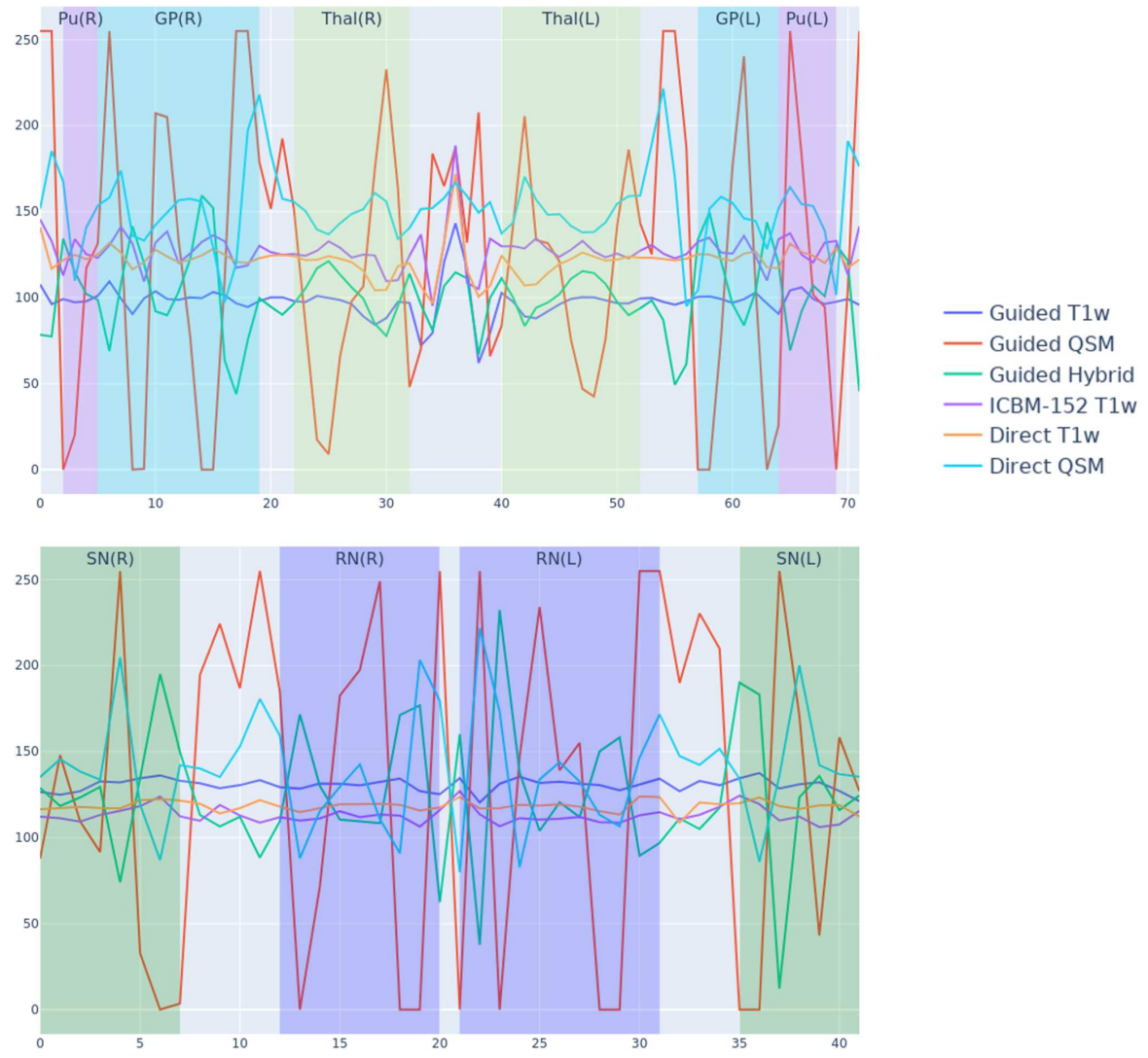

Fig. 5 Intensity distribution along profile lines across patches containing the basal ganglia and thalamus. The voxel intensities of the profile lines as shown in Figure 4 enlarged views are plotted to demostrate image contrast in the ROI.

atlas are as sharp as the T1w atlases, and the typical features of DBN on QSM atlas are preserved on the hybrid atlas, such as the red nucleus and thalamic subnuclei.

According to the profile lines shown in Figure 5, same modalities presents similar level of image contrast. The profile line of the QSM atlas shows the great sharpness, indicated by the largest intensity variations (0 to about 252), and thus retains the most clear features of the DBN anatomical structures. The profile lines of both $\mathrm{T} 1 \mathrm{w}$ atlases show much smaller variation (about 105 to about 150), although with a similar variation trend as in the QSM profile line. The profile lines of the hybrid atlas show medium variation (about 40 to 200). Notably, the line of hybrid atlas properly combines both T1w and QSM atlas features, showing the same trend with $\mathrm{T} 1 \mathrm{w}$ atlas and retaining the twists and turns of the QSM atlas.

The result of k-means intensity clustering on a selected slice is shown in Figure 6, where the generated rough parcellation maps is overlapped with 
the tissue intensity image. We set $k=4$ as the optimal parameter after multiple attempts. For the guided atlases, the worst segmentation is observed on the $\mathrm{T} 1 \mathrm{w}$ atlases, where the gray matter and white matter tissues can not be accurately separated. The segmentations on the two QSM atlases accurately delineate the typical DBN, such as the $\mathrm{Cu}$ and $\mathrm{Pu}$, and the thalamus is divided into two parts. However, the white matter tissue and cerebrospinal fluid can not be accurately segmented on the QSM atlas. The segmentation result of the hybrid atlas outperforms those of the T1w and QSM atlases, where the gray matter is more accurately delineated from the surrounding white matter tissue and cerebrospinal fluid. Furthermore, the delineation of DBN on the hybrid atlas is finer than that on the T1w atlas. Similar segmentation performance compared with those of guided T1w and QSM atlases can be observed on the directed T1w and QSM atlases.

\subsection{DBN parcellation map}

Both the original atlases and the border-enhanced display of the multi-modal atlases (the T1w and QSM atlases) are referred to for the parcellation of the DBN, so that we could make full use of the features on the multi-modal atlases.

Figure 7 shows multi-modal atlases where delineation of the nuclei of interest are referred. 3 typical slices with multi-modal atlases are shown in group, in order to clarify which image modal is preferred in the manual delineation of different DBN. Meanwhile, manual delineated curve is overlapped on top of the evidencing boundary in nuclei delineation (single hemisphere only for slices in axial view). Note that the curve of each nuclei is delineated on the exact referred atlas in nuclei delineation step, and directly translated to the intra-group atlas samples. For example, the $\mathrm{Pu}$ was directly delineated on the border-enhanced display of T1w atlas (Figure 7a). The GP (external \& internal) are delineated on the original QSM atlas since the borderline is not with sharp enough contrast on the T1w or the border-enhanced QSM displays (Figure $7 \mathbf{b}$, blue \& red). The $\mathrm{Ca}$ and NAcc are depicted on the sagittal view of the original T1w atlas, for only the T1w atlas most clearly shows the NAcc. The Ca is firstly depicted with reference to the NAcc, as the NAcc is positioned to the inner boundary of the Ca (Figure 7c). Depiction of other nuclei in the basal ganglia, including the RN, SN and STN, is performed on the original QSM atlas since the border-enhanced scan is unable to provide more competitive contrast (Figure 7c). The STN is delineated on the original QSM atlas. The SNr and SNc are depicted on both T1w and QSM atlases since the outer border of the $\mathrm{SNr}$ and the inner border of the $\mathrm{SNc}$ are inconsistent across modalities. To solve the inconsistency, the $\mathrm{SNr}$ was firstly depicted with reference to the position of the STN, and the SNc is then depicted with reference to the position of the SNr. The red nucleus is depicted on the QSM atlas.

Figure 8 shows the DBN parcellation result, including a 3-D rendering view (upper panel) and 2-D slice views covered on the constructed T1w atlas (lower panel). The zoomed-in view of the regions of interest are also shown for 


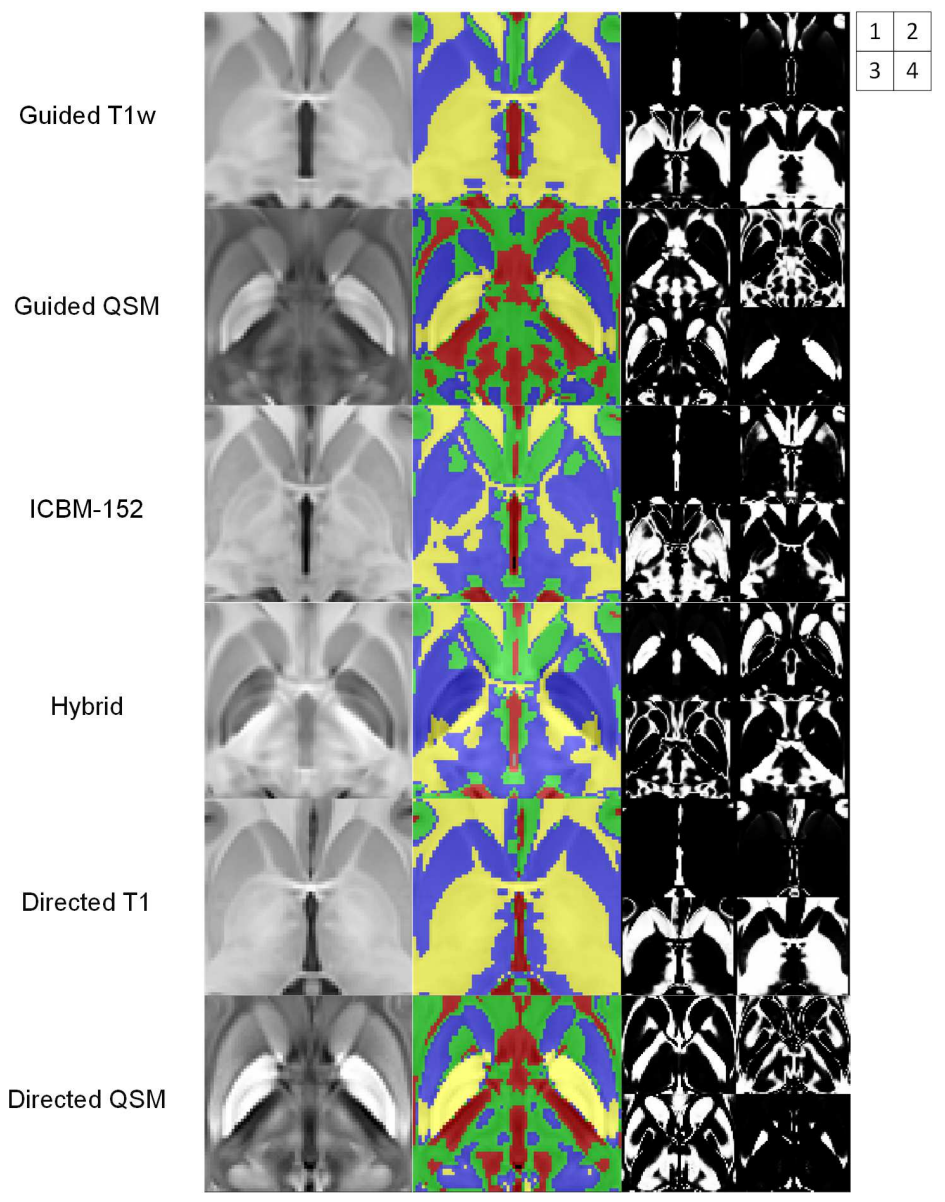

Fig. 6 K-means intensity clustering result overlapped on the patch containing the basal ganglia and thalamus of the hybrid-guided and the directly generated atlases. From the left to the right columns: the original patches, segmented tissues overlaid on patches, and binary-valued images of the four segmented tissues.

closer visualization. The volumes of the nuclei calculated from the parcellation are shown in Table 1.

\subsection{Accuracy of using parcellation map to delineate DBN in individual images}

The comparison results for assessing atlas-guided DBN segmentation accuracy in the individual QSM images are shown at Figure 9. Our atlas performs better than the mentioned previous atlases by $5 \%$ to $10 \%$ in both evaluation metrics (Dice coefficient and Hausdorff distance) in each group. The averaged Dice coefficients of our parcellation are higher than 0.8 (some even higher than 0.9 ), ranging from 0.73 to 0.90 . In contrast, the previous atlases mostly conduct Dice coefficients lower than 0.8 , ranging from 0.6 to 0.7 . The Hausdorff distance 


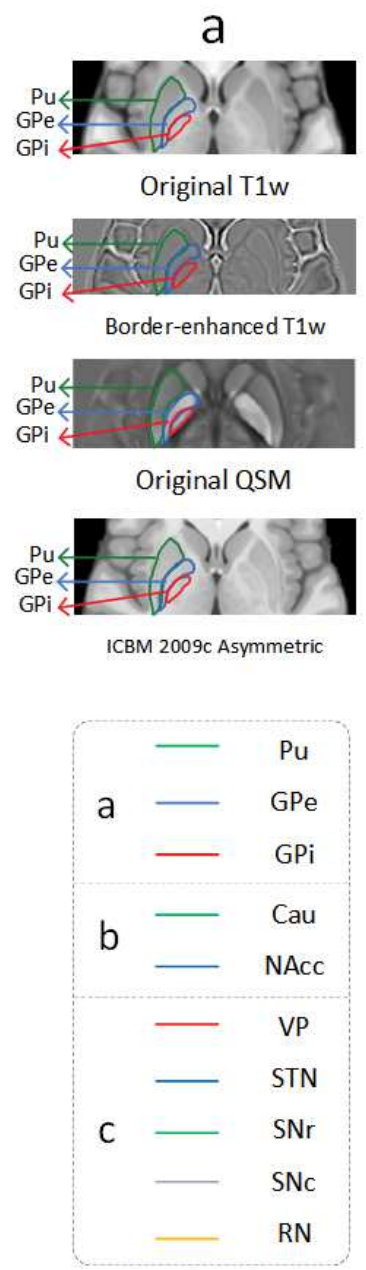

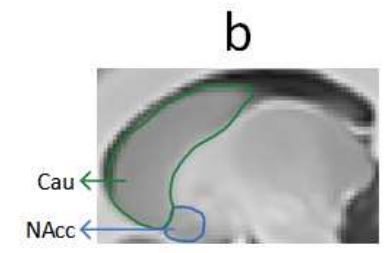

Original T1w

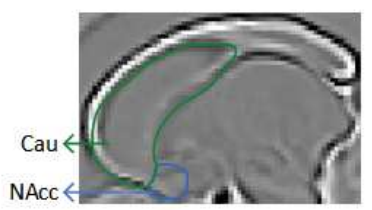

Border-enhanced T1w

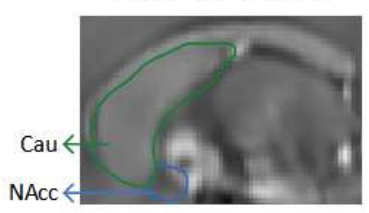

Original QSM

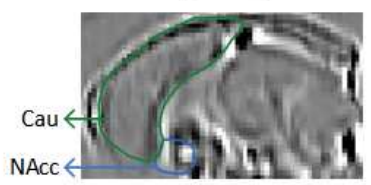

Border-enhanced QSM

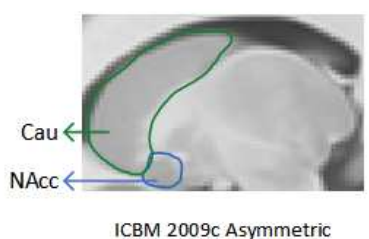

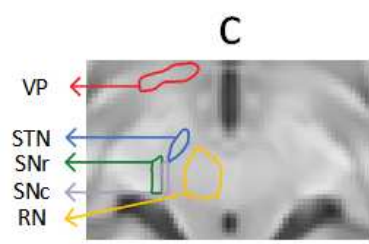

Original T1w

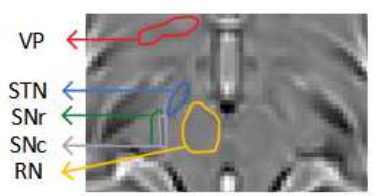

Border-enhanced T1w

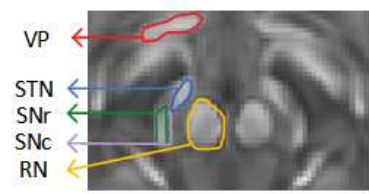

Original QSM

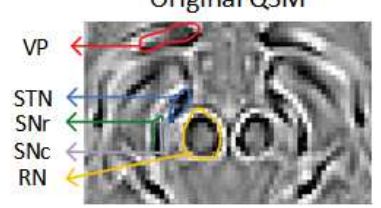

Border-enhanced QSM

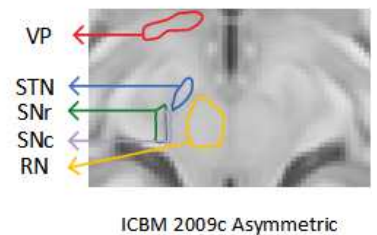

Fig. 7 Multi-view illustration of contextual neuroimaging depiction of delineation for nuclei of interest in MNI-space atlases. Sampled slices of multi atlases along with manual delineation of the nuclei of interest are grouped in 3 columns. Note that the delineation samples of a group are identical, where the delineation is directly translated onto the corresponding location of the remaining scans without without transformation. (a) $\mathrm{Pu}, \mathrm{GPe}$ and GPi displayed in axial view. (b) $\mathrm{Ca}$ and NAcc displayed in sagittal view. (c) $\mathrm{VP}, \mathrm{STN}, \mathrm{SNr}, \mathrm{SNc}$ and RN displayed in axial view.

also suggests a better outcome of DBN delineation using our parcellation than using the previous parcellations, as indicated by lower average distance and more stable results among the individuals. In addition, our atlas produces fewer accidental errors, with fewer individual outliers compared with the other atlases.

For the coarse-parcellation group, our delineation of all the nuclei achieves higher Dice coefficients compared with that of the previous atlases. The Dice 


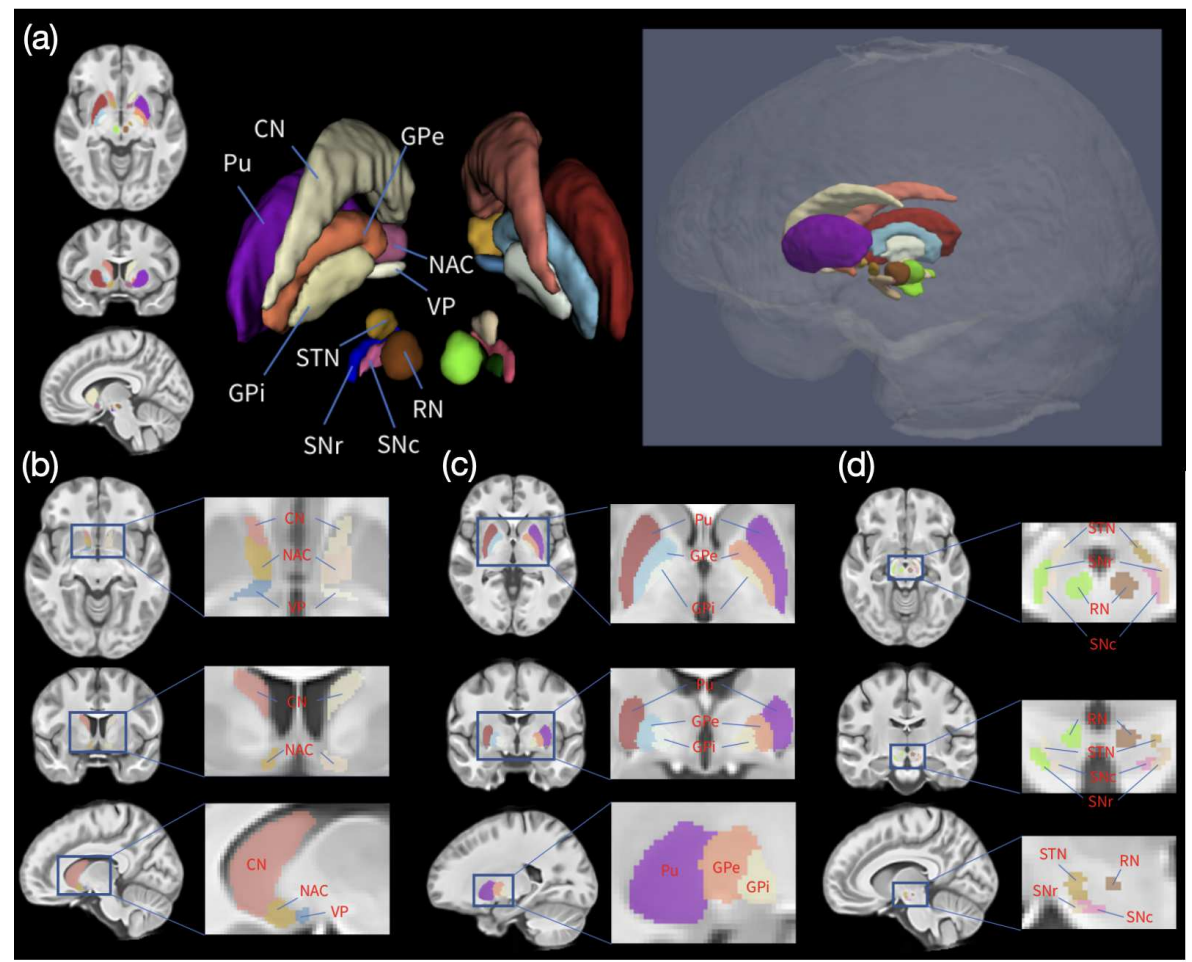

Fig. 8 The manually annotated parcellation result based on the constructed multi-modal atlas in MNI space. (a) A general view of the map shown in a single chosen slice. (b) 3-D rendering of DBN with labels. (c) 3-D rendering of DBN placed in the whole brain. (d) Three views of the parcellations of basal ganglia nuclei.

Table 1 Volumes of depicted DBN $\left(\mathrm{mm}^{3}\right)$

\begin{tabular}{|c|c|c|c|c|c|c|c|c|c|}
\hline \multirow{2}{*}{\multicolumn{2}{|c|}{ Cau }} & \multicolumn{2}{|c|}{$\mathrm{Pu}$} & \multicolumn{4}{|c|}{ GP } & \multicolumn{2}{|c|}{ NAcc } \\
\hline & & & & \multicolumn{2}{|c|}{ GPe } & \multicolumn{2}{|c|}{ GPi } & & \\
\hline $\mathrm{L}$ & $\mathrm{R}$ & $\mathrm{L}$ & $\mathrm{R}$ & $\mathrm{L}$ & $\mathrm{R}$ & $\mathrm{L}$ & $\mathrm{R}$ & $\mathrm{L}$ & $\mathrm{R}$ \\
\hline 4593 & 4507 & 6137 & 5853 & 2037 & 1967 & 616 & 569 & 329 & 329 \\
\hline \multicolumn{2}{|c|}{ VP } & \multicolumn{2}{|c|}{ STN } & \multicolumn{4}{|c|}{ SN } & \multicolumn{2}{|c|}{$\mathrm{RN}$} \\
\hline & & & & & & & & & \\
\hline $\mathrm{L}$ & $\mathrm{R}$ & $\mathrm{L}$ & $\mathrm{R}$ & $\mathrm{L}$ & $\mathrm{R}$ & $\mathrm{L}$ & $\mathrm{R}$ & $\mathrm{L}$ & $\mathrm{R}$ \\
\hline 128 & 134 & 107 & 111 & 142 & 130 & 90 & 73 & 285 & 293 \\
\hline
\end{tabular}

coefficients are high with small variance for the GP, Pu, Cau and RN. The Dice scores of SN and NAcc are also higher than the other atlases. Our atlas also shows a relatively shorter Hausdorff distance than Zhang's and AAL3 atlases, with lower mean value and variance (e.g., the distance of our results versus that of Zhang's atlas). Both of the other atlases show suboptimal results for bilateral Cau, while our atlas achieves a low mean in the distance with slightly high variance, compared with significantly higher means in the distance of the 
other atlases. Notice that NAcc is not included in Zhang's atlas, and thus the corresponding results of both metrics are presented as 0 .

For the comparison of the high-resolution group, the delineation using our parcellation outperforms AHEAD atlas from both Dice coefficient and Hausdorff distance perspectives with better mean value and lower variance. For difficult nuclei such as STN and SN, our atlas still shows competitive performance with both reasonable value and variance not out of the ordinary.

\subsection{Result and analysis of fine parcellation in thalamus}

Figure 10 shows the axial and coronal views of the manually annotated thalamic sub-nucleus parcellation map overlays on a high-resolution (7T) QSM image, with the histology reference shown on the right side. The QSM image with a voxel resolution of $0.4 \times 0.4 \times 0.4 \mathrm{~mm}^{3}$ provides clear contrast among thalamic sub-nuclei for annotation. Table $\mathbf{2}$ (shown in the appendix) presents the list of the total 32 sub-nuclei of the thalamus on each side and the corresponding color codes. Figure 11 illustrates two representative slices of the $3 T$ QSM atlas with a fused parcellation map overlays on the top, with the histology reference shown on the right side. The fused sub-nucleus labels in standard MNI space of thalamus shows more smooth bounderies than that in the individual 7T guidance image. The label fusion strategy facilitate histologyconsistent thalamus sub-nucleus annotation in the atlas space. In the final parcellation map, we can precisely identify crucial thalamic sub-nucleus such as the Ventrointermedius (V.i.m., highly related to PD), Dorsomedialis (M, related to schizophrenics), Anteroprincipalis (A.pr, a vital component of the hippocampal system for episodic memory).

\subsection{DBN with PD-related abnormality in iron content identified on the atlas}

The map revealing the group differences in brain tissue susceptibility between the PD patients and the normal controls indicated by voxel-wise unpaired t-test is shown in Figure 12 using ITK-SNAP (Yushkevich et al., 2006). The voxels with FWE-corrected $P$-values less than 0.05 are overlaid on the MNI-spaced QSM atlas. For focusing attention on DBN regions, we mask the non-DBN susceptibility variations out of our significance map. The boundaries of DBN are further overlaid on the significance map to identify the DBN with disease-related alternations in iron content. Figure 13 shows a representative slice with detailed DBN parcellation. The abnormalities in iron content are found in the bilateral $\mathrm{Ca}, \mathrm{Pu}, \mathrm{GP}$, Thal, RN, as well as the left STN nucleus and SN (both SNr \& SNc). The volume of regions with abnormality in QSM in the right hemisphere is slightly larger than that in the left hemisphere.

The abnormality in the thalamus mainly distributes from the ventral region to the central medial region, approximately from the coordinate of $-5 \mathrm{~mm}$ to $13 \mathrm{~mm}$ along the Z-axis, shaped like a spindle with its most part on the ventral side. Here we report the subregions of the thalamus with an occupation 
Dice coefficient (coarse group)

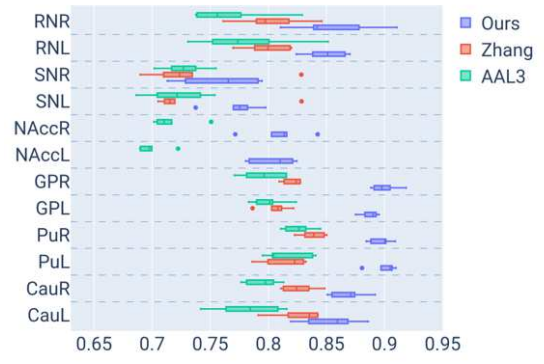

Dice coefficient (fine group)

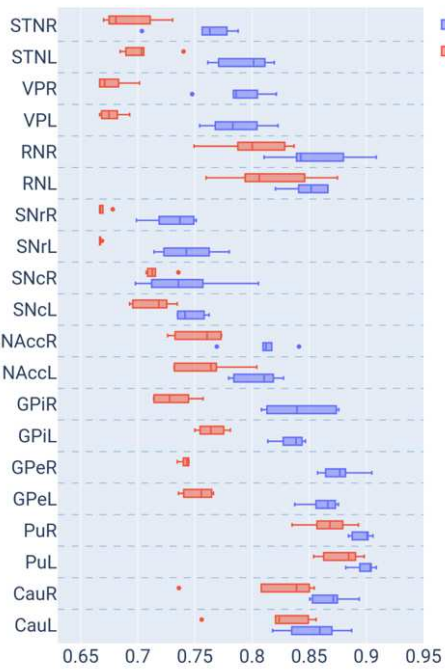

Dice coefficient (high-resolution group)

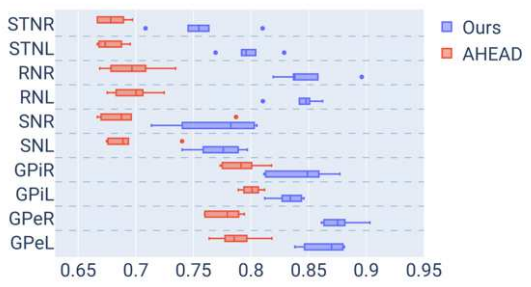

Hausdorff distance (coarse group)

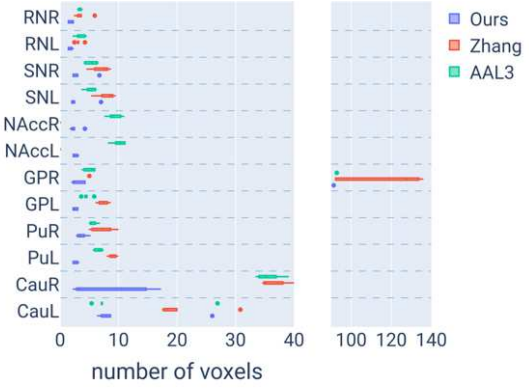

Hausdorff distance (fine group)

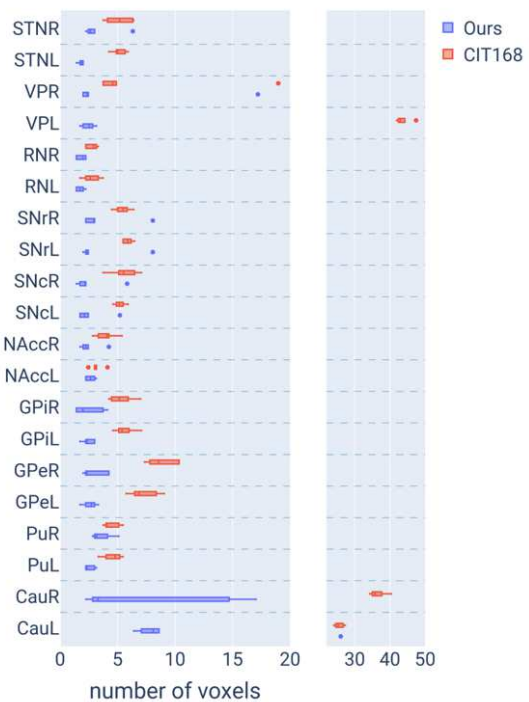

Hausdorff distance (high-resolution group)

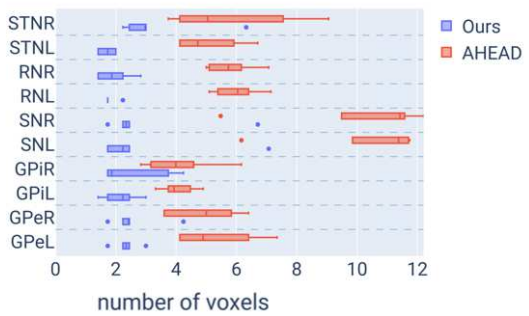

Fig. 9 Atlas-guided DBN segmentation performance evaluation of label propagation in individuals. The top, middle, and bottom rows show the comparison results for the coarse group, fine group, and high-resolution group respectively. The left and right columns show the results of the Dice coefficient and Hausdorff distance respectively.

rate of significant voxels more than $30 \%$ of the total voxels within the subregions. The thalamic subregions identified with significantly abnormal iron 


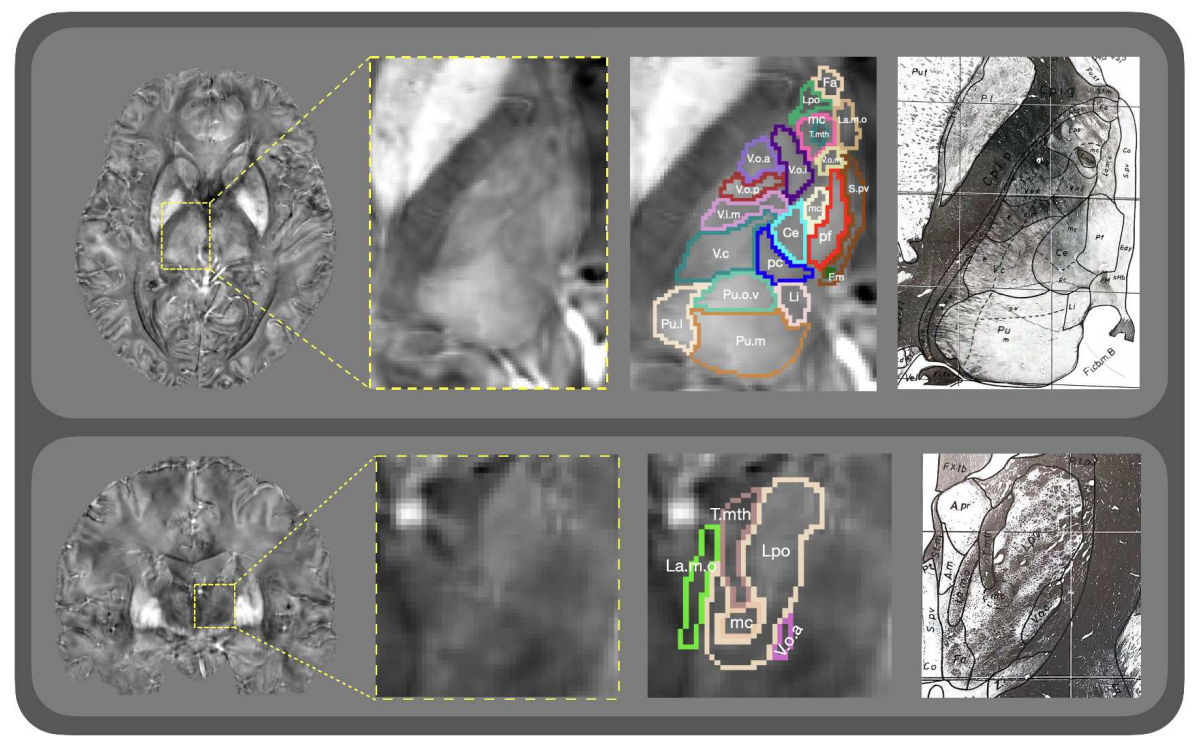

Fig. 10 Parcellation map of thalamic subnuclei on a 7T QSM image. The segmentation labels are overlaid on an axial slice (upper row) and a coronal slice (lower row) views of the 7T QSM image with the Schaltenbrand and Wahren histology atlas reference on the right.

content in $\mathrm{PD}$, ranked by the occupation rate, include the centralis lateralis (R, 94.595\% \& L, 50.000\%), paracentral (R, 93.462\% \& L, 68.803\%), centralis thalami (R, 78.947\% \& L, 62.234\%), ventrointermedius ( $\mathrm{R}, 68.696 \%)$, ventrooralis posterior $(\mathrm{R}, 64.671 \%$ ), dorso-caudalis ( $\mathrm{L}, 63.316 \%$ \& $\mathrm{R}, 60.324 \%$ ), ventrooralis internus ( $\mathrm{L}, 62.766 \%$ \& $\mathrm{R}, 62.587)$, fasciculus retroflexus Meynertii (R, 57.143\%), dorso-intermedii (L, 47.264\%), ventrocaudalis ( $\mathrm{R}, 43.740 \%)$, mc in Lpo (L, 40.000\% \& R, 35.294\%), ventrooralismedialis ( R, 39.823\% \& L, $29.921 \%$ ), parafascicularis thalami (L, 35.067\%), and pulvinaris superficialis $(\mathrm{R}, 31.068 \%)$. The results show a generally symmetric manner of susceptibility elevation in all mentioned thalamic subregions in the PD patients, as nearly all the corresponding subregions on the left and right show similar occupation rates of significant voxel numbers. As mentioned, the ventral and the central subregions of the thalamus show the highest occupation rates from the group-wise comparison.

Our results are consistent with the previous reports of the PD-related alterations in iron content. For example, a study using the voxel-wise permutation test revealed QSM abnormality in the SN and STN (Acosta-Cabronero et al., 2016). The abnormality in SN is consistently found in PD patients as revealed by a systematic review that reported 30 out of 33 (90.9\%) studies indicated significant PD-related QSM abnormality in the SN (Ravanfar et al., 2021). This review also suggested a wide range of brain areas, including the $\mathrm{Ca}, \mathrm{Pu}$, and Thal, with higher susceptibility in PD patients with cognitive impairments. 


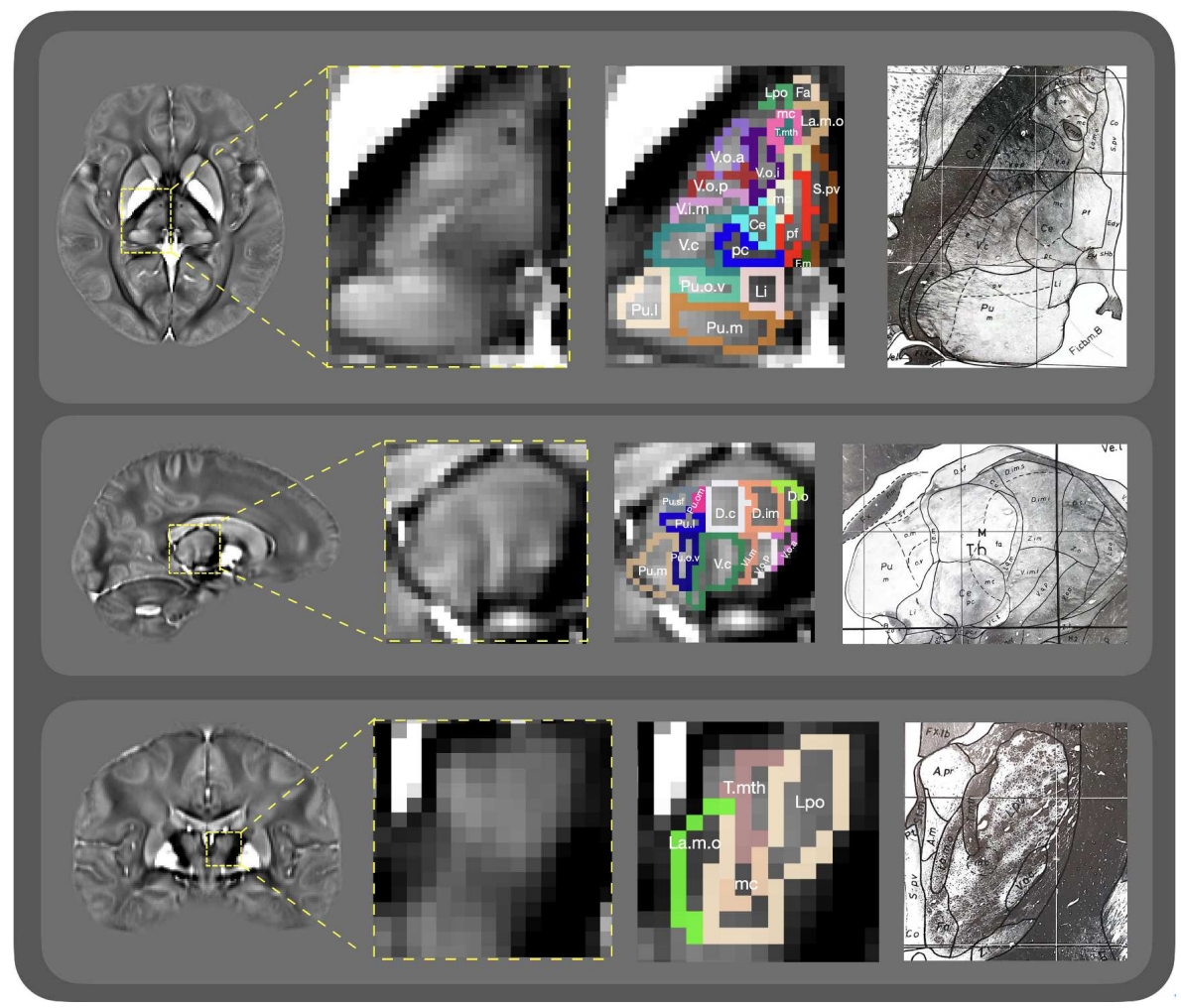

Fig. 11 Thalamic parcellation map on three representative slices of our 3T atlas. The thalamic subnuclei labels are overlaid on the axial (top row), sagittal (middle row), and coronal (bottom row) slice views. The Schaltenbrand and Wahren histology references are shown on the right.

\section{Discussions}

In the present study, we firstly introduced the MuSus-100 hybrid image guided MNI-space QSM atlas construction and the DBN parcellation based on these atlases. Quantitative analysis indicates that the T1w-QSM hybrid atlas better depicts the cortical and subcortical structures than the T1w atlas or QSM atlas alone. Furthermore, the MuSus-100 atlas outperforms the current existing atlases in accuracy and fineness of DBN segmentation in individual images. In addition, a fine parcellation of the thalamic subnuclei is introduced via a label fusion strategy to our 3.0-Tesla atlas using a manual parcellation on 7.0-Tesla QSM image as guidance.

\subsection{Quality of constructed atlases and parcellation maps}

Our atlases were constructed from 100 young adult volunteers with age ranging in 19-29. The age distribution is quite close to that of Colin 27 (28-years individual) (Holmes et al., 1998) and ICBM 152 (18.5-43.5) (V. Fonov et al., 2011). 


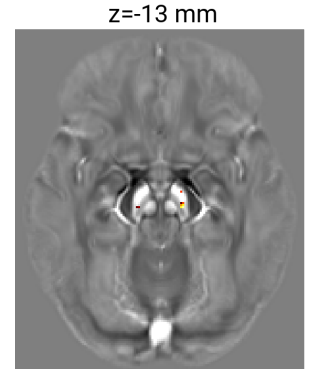

$\mathrm{z}=7 \mathrm{~mm}$

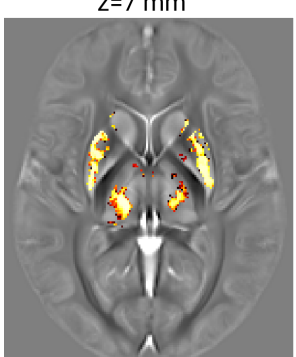

$\mathrm{z}=-8 \mathrm{~mm}$

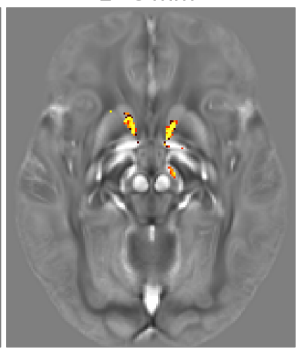

$\mathrm{z}=12 \mathrm{~mm}$

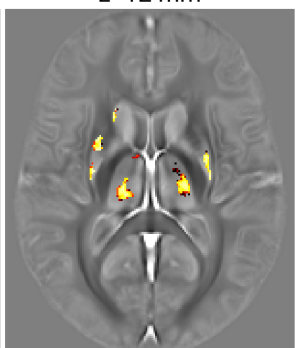

$\mathrm{z}=-3 \mathrm{~mm}$

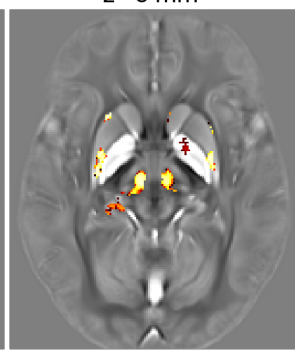

$\mathrm{z}=17 \mathrm{~mm}$

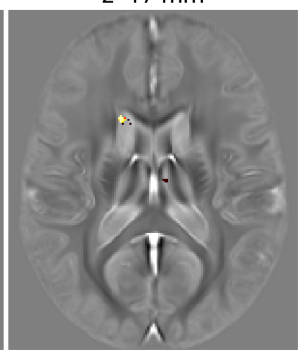

$\mathrm{z}=2 \mathrm{~mm}$

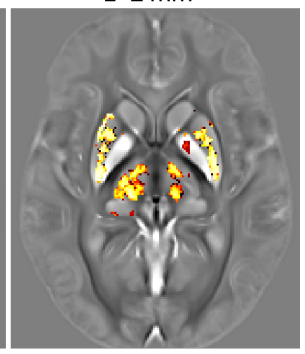

$\mathrm{z}=22 \mathrm{~mm}$

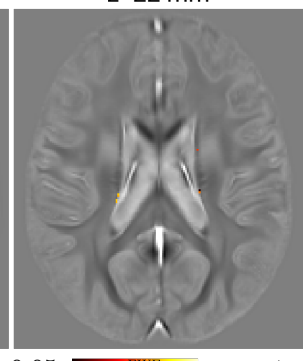

0.05

$2 \times 10^{-4}$

Fig. 12 Significance map $\left(P^{\mathrm{FWE}}<0.05\right)$ revealing the difference in tissue susceptibility between PD patients and healthy controls indicated by a voxel-wise permutation test with age-correction. The between-group comparison heat map result is overlaid on the constructed MNI-spaced QSM atlas.

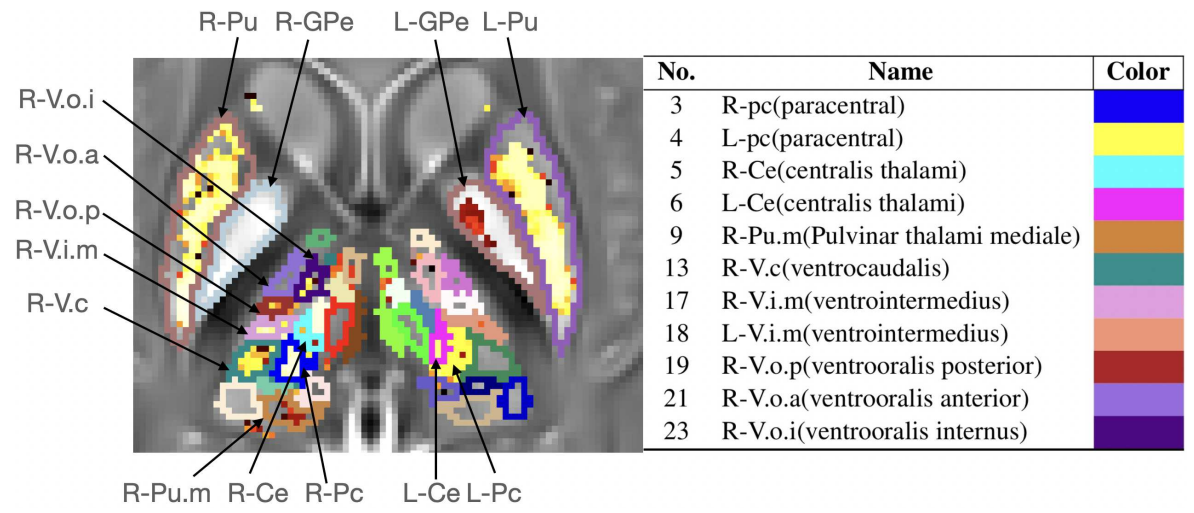

Fig. 13 A representative slice of QSM atlas with significance map $\left(P_{F W E}<0.05\right)$ and DBN boundaries overlaid on top. The nuclei with extensive coverage of significant voxels are indicated by arrows. The names of thalamic subregions are shown in the sheet displayed on the right.

With similar age distribution, the brain image quality and brain structure are also similar between each dataset, thus resulting potential easier initialization for spatial normalization between our constructed T1w atlas and MNI space T1w atlas. The image registration and projection were well controlled by introducing suitable processing steps, including the hybrid image guided 
group-wise registration, the average template sharpening update, and the $\mathrm{T} 1 \mathrm{w}$ atlas guided MNI-space projection. In particular, the hybrid image combines the advantages of both the T1w image and the QSM image, which results in a more accurate registration in both the cortical and subcortical structures across the samples.

Both visual inspection and contrast analysis demonstrate high quality for the constructed atlases in the delineating the DBN of interest. By direct visual inspection, several small size DBN and the DBN anatomical sub-regions can be clearly identified, which are usually invisible or not clear in other atlases. For instance, the division of GPe and GPi can be observed even in our T1w atlas. We performed the k-means intensity clustering on selected image patches, and the border-enhanced displays to assess the image quality for constructed atlas. Both analyses show that the QSM atlas provides a sharp contrast in DBN regions, which improve the delineation performance for nuclei of interest. In addition, both the constructed T1w and QSM atlases and the border-enhanced display are considered as references to maximally improve the accuracy of DBN parcellation.

\subsection{Accuracy of the atlas-based DBN parcellation of individual images}

The results show that our atlas outperforms those of the existing DBN parcellations based on the other T1w and QSM atlases both in the accuracy and stability on the work of propagating the parcellation map to the individual QSM images. For all nuclei, the delineation generated by our parcellation outperforms those for comparison in both mean and variance, which indicates that our result provides higher accuracy and stability. Since the difficulty of the depiction of nuclei may vary due to different local neuroimaging contrast and inter-individual variations, the nuclei easier to depict may achieve higher accuracy and stability. In our present study, the GP, RN, Cau, VP are relatively easy to depict for they have sharp boundaries, while the STN, SN, and NAcc are relatively hard to depict due to the coarse boundary and fuzzy local features.

For those difficult DBN, the performance of both our parcellation and those based on the atlases for comparison in delineating DBN in the individuals were not ideal. The propagation of these atlases results in relatively low mean Dice coefficient scores. Moreover, the outliers in the result of Hausdorff distance are more likely to occur for these nuclei. Nevertheless, our atlas still outperforms those for comparison, with fewer outliers and equivalent Dice coefficient and Hausdorff distance scores for the nuclei difficult to depict. Thus, our atlas still provides relatively satisfying results in the nuclei with fuzzy surrounding information. 


\subsection{Application prospects of the atlases and parcellation maps}

Accurate and fine parcellations of subcortical nuclei, particularly the nuclei within the basal ganglia and thalamus, are greatly needed in the studies of neurological and psychiatric. Specific types of symptoms of neurological and psychiatric diseases may be associated with abnormal structural or functional features in specific DBN. Furthermore, deep brain stimulation (DBS) targeting the DBN has been used to treat neurological and neuropsychiatric disorders in patients who could not receive sufficient effect from non-invasive therapies (Williams \& Okun, 2013). Studies examining the relationship between the anatomical location of DBS and the treatment effect have been carried out recently to identify the optimal location of stimulation (Bot et al., 2018; Matsumoto et al., 2016). Thus faithful depiction of DBN based on neuroimaging is crucial for the studies of disease pathology and target refining for deep brain stimulation.

Our atlases derived from healthy young volunteers (around 25 years old) may serve as a representation of a healthy young sample to be compared with samples with older ages in the longitudinal studies on QSM alterations (Peterson et al., 2019; Ward et al., 2019; Zucca et al., 2006). For example, Zhang et al. (2018) performed longitude analysis on the quantitative susceptibility changes using fine labeled QSM and T1w scans. The individual scans are registered into a single $3 \mathrm{~T}$ atlas with a parcellation map depicting iron-rich DBN. The DBN in individual scans can be delineated by applying inverse warp of the transformation field of the registration, and thus the magnetic susceptibility and iron-depositing volumes of regions of interest can be calculated.

\subsection{Mapping of thalamus subnuclei and its application prospects}

As reported by a series of recent studies, QSM based on the GRE sequence is superior in delineating the thalamic subnuclei (Deistung et al., 2013; He et al., 2020; J. Li et al., 2019). Here we for the first time provide a fine parcellation of the thalamic subnuclei based on QSM from a 7T MRI, which identifies 32 subnuclei of the thalamus on each side confirmed with the Schaltenbrand and Wahrn histology atlas. Due to the high expense of manual segmentation, we manually delineated the thalamic subnuclei on only one $7 \mathrm{~T}$ image as the ground truth. This might introduce individual bias. To solve this issue, we projected the parcellation on to all the $3 \mathrm{~T}$ individual images by non-linear registration, and afterwards fused the individual parcellations in the atlas space to achieve the final parcellation of thalamic subnuclei. Such a process should be able to guarantee our parcellation unbiased.

Using the fine parcellation map of the thalamus, we can precisely identify critical thalamic subnuclei such as the Ventrointermedius (VIM, highly related to tremor), Dorsomedialis (M, related to schizophrenics), and Anteroprincipalis (A.pr, a vital component of the hippocampal system for episodic 
memory). Thus, the proposed thalamic parcellation map in standard space can become a powerful tool for understanding related neurological disease progression and providing insights for the treatments of diseases related to abnormalities. This fine atlas of thalamic subnuclei could also provide a visual reference to confirm the accuracy of indirect targeting, which may potentially help to improve treatment effect of the surgeries of thalamic subnuclei. Meanwhile, in neurosurgery experiences, the purposed thalamic parcellation map allows positioning for critical targets in DBS surgeries using T1 and T2 scans. And the fine parcellation also boosts researches on subthalamic connectomics, which offers fine-targeted ROIs for finding the relationship between related diseases and the abnormal ROI connections in a more precious view in data-abundant 3T space.

\section{Conclusions}

In the present study, we introduce a MNI-spaced human brain QSM atlas and parcellation map, named MuSus-100 atlas. The QSM atlas is derived from the T1w, QSM, and GRE magnitude images of 100 healthy young volunteers via group-wise registration guided by the T1w-QSM linear hybridization of the individual images. The hybrid image highlights the representation of subcortical nuclei, and meanwhile, preserves the advantages of clear anatomical tissue structure in T1w images. The constructed MNI-space QSM atlas provides sharp contrast for facilitating the delineation for the DBN of interest. Eleven highly interested DBN were depicted based on atlases of multiple modalities. In addition, a fine thalamus parcellation map based on the $7 \mathrm{~T}$ individual QSM was introduced into MNI-space, providing a detailed histology-consistent thalamic subnucleus parcellation for MNI-spaced images acquired from a 3T scanner. The constructed parcellation maps clearly delineate DBN of interest in the standard MNI space, which can help the ROI-based quantitative analysis on iron-rich subcortical nuclei in future studies. The proposed atlas has the potential value to facilitate the research in the field of neurological and psychiatric disease progress and DBS surgical target refining.

\section{Disclosures and declarations}

Data availability statement: Materials to be released include the proposed multi-modal MuSus-100 atlases (T1w atlas, QSM atlas) and the codes for atlas constructing and experiments. However, due to further censoring and uploading to server, the materials are not available yet. The materials are about to be released on later 2021 online, including the link of cloud storage (for atlases) and GitHub repo (for codes).

Funding statement: This study is supported by the National Natural Science Foundation of China (No. 62071299, 61901256, 91949120); Xiaojun Guan is supported by the National Natural Science Foundation of China (Grant No. 82001767), the Natural Science Foundation of Zhejiang Province (Grant No. 
LQ21H180008) and the China Postdoctoral Science Foundation (Grant No. 2021T140599 and 2019M662082).

Conflict of interest: The authors declare that the research was conducted in the absence of any commercial or financial relationships that could be construed as a potential conflict of interest.

Ethics approval statement: The authors are accountable for all aspects of the work in ensuring that questions related to the accuracy or integrity of any part of the work are appropriately investigated and resolved. All procedures of the present study are in accordance with the Declaration of Helsinki, and are approved by the Ethics Committee of the Second Affiliated Hospital, Zhejiang University School of Medicine.

Patient consent statement: The participants are informed of the study procedures and all give their written consent.

Permission to reproduce material from other sources: No materials are reproduced from other sources and no statement is needed.

Clinical trial registration: No clinical trial operations are involved in this research.

\section{References}

Acosta-Cabronero, J., Cardenas-Blanco, A., Betts, M.J., Butryn, M., Valdes-Herrera, J.P., Galazky, I., Nestor, P.J. (2016, 11). The whole-brain pattern of magnetic susceptibility perturbations in Parkinson's disease. Brain, 140(1), 118-131. Retrieved from https://doi.org/10.1093/brain/aww278 https://arxiv.org/abs/https:// academic.oup.com/brain/article-pdf/140/1/118/23001949/aww278.pdf $10.1093 /$ brain/aww278

Alkemade, A., Mulder, M.J., Groot, J.M., Isaacs, B.R., van Berendonk, N., Lute, N., .. Forstmann, B.U. (2020). The amsterdam ultra-high field adult lifespan database (ahead): A freely available multimodal 7 tesla submillimeter magnetic resonance imaging database. NeuroImage, 221, 117200 .

Avants, B.B., Epstein, C.L., Grossman, M., Gee, J.C. (2008). Symmetric diffeomorphic image registration with cross-correlation: evaluating automated labeling of elderly and neurodegenerative brain. Medical image analysis, 12(1), 26-41.

Avants, B.B., Tustison, N., Song, G. (2009). Advanced normalization tools (ants). Insight $j, 2(365), 1-35$. 
Behrens, T.E., Johansen-Berg, H., Woolrich, M., Smith, S., WheelerKingshott, C., Boulby, P., ... others (2003). Non-invasive mapping of connections between human thalamus and cortex using diffusion imaging. Nature neuroscience, 6(7), 750-757.

Betts, M.J., Acosta-Cabronero, J., Cardenas-Blanco, A., Nestor, P.J., Düzel, E. (2016). High-resolution characterisation of the aging brain using simultaneous quantitative susceptibility mapping (qsm) and $\mathrm{r} 2^{*}$ measurements at 7 t. Neuroimage, 138, 43-63.

Bilgic, B., Pfefferbaum, A., Rohlfing, T., Sullivan, E.V., Adalsteinsson, E. (2012). Mri estimates of brain iron concentration in normal aging using quantitative susceptibility mapping. Neuroimage, 59(3), 2625-2635.

Bot, M., Schuurman, P., Odekerken, V., Verhagen, R., Contarino, F., De Bie, R., van den Munckhof, P. (2018). Deep brain stimulation for parkinson's disease: defining the optimal location within the subthalamic nucleus. $J$ Neurol Neurosurg Psychiatry, 89(5), 493-498.

Butson, C.R., Cooper, S.E., Henderson, J.M., McIntyre, C.C. (2007). Patientspecific analysis of the volume of tissue activated during deep brain stimulation. Neuroimage, 34(2), 661-670.

Deistung, A., Schäfer, A., Schweser, F., Biedermann, U., Turner, R., Reichenbach, J.R. (2013). Toward in vivo histology: a comparison of quantitative susceptibility mapping (qsm) with magnitude-, phase-, and r $2 *$-imaging at ultra-high magnetic field strength. Neuroimage, 65, 299-314.

Eisenstein, S.A., Koller, J.M., Black, K.D., Campbell, M.C., Lugar, H.M., Ushe, M., . . others (2014). Functional anatomy of subthalamic nucleus stimulation in parkinson disease. Annals of neurology, 76 (2), 279-295.

Ewert, S., Plettig, P., Li, N., Chakravarty, M.M., Collins, D.L., Herrington, T.M., .. Horn, A. (2018). Toward defining deep brain stimulation targets in mni space: a subcortical atlas based on multimodal mri, histology and structural connectivity. Neuroimage, 170, 271-282.

Fonov, V., Evans, A.C., Botteron, K., Almli, C.R., McKinstry, R.C., Collins, D.L., ... others (2011). Unbiased average age-appropriate atlases for 
pediatric studies. Neuroimage, 54(1), 313-327.

Fonov, V.S., Evans, A.C., McKinstry, R.C., Almli, C., Collins, D. (2009). Unbiased nonlinear average age-appropriate brain templates from birth to adulthood. NeuroImage(47), S102.

Haacke, E., Tang, J., Neelavalli, J., Cheng, Y. (2010). Susceptibility mapping as a means to visualize veins and quantify oxygen saturation. Journal of Magnetic Resonance Imaging, 32(3), 663-676.

Hanspach, J., Dwyer, M.G., Bergsland, N.P., Feng, X., Hagemeier, J., Bertolino, N., ... Schweser, F. (2017). Methods for the computation of templates from quantitative magnetic susceptibility maps (qsm): Toward improved atlas-and voxel-based analyses (vba). Journal of Magnetic Resonance Imaging, 46(5), 1474-1484.

He, N., Langley, J., Huddleston, D.E., Ling, H., Xu, H., Liu, C., ... Hu, X.P. (2017). Improved neuroimaging atlas of the dentate nucleus. The Cerebellum, 16(5-6), 951-956.

He, N., Sethi, S.K., Zhang, C., Li, Y., Chen, Y., Sun, B., .. Haacke, E.M. (2020). Visualizing the lateral habenula using susceptibility weighted imaging and quantitative susceptibility mapping. Magnetic Resonance Imaging, 65, 55-61.

Holmes, C.J., Hoge, R., Collins, L., Woods, R., Toga, A.W., Evans, A.C. (1998). Enhancement of $\mathrm{mr}$ images using registration for signal averaging. Journal of computer assisted tomography, 22(2), 324-333.

Horn, A., \& Kühn, A.A. (2015). Lead-dbs: a toolbox for deep brain stimulation electrode localizations and visualizations. Neuroimage, 10\%, 127-135.

Klein, A., \& Tourville, J. (2012). 101 labeled brain images and a consistent human cortical labeling protocol. Frontiers in neuroscience, 6, 171.

Langkammer, C., Krebs, N., Goessler, W., Scheurer, E., Ebner, F., Yen, K., .. . Ropele, S. (2010). Quantitative mr imaging of brain iron: a postmortem validation study. Radiology, 257(2), 455-462. 
Lau, J.C., Xiao, Y., Haast, R.A., Gilmore, G., Uludağ, K., MacDougall, K.W., ... Khan, A.R. (2020). Direct visualization and characterization of the human zona incerta and surrounding structures. Human brain mapping, 41(16), 4500-4517.

Lenglet, C., Abosch, A., Yacoub, E., De Martino, F., Sapiro, G., Harel, N. (2012). Comprehensive in vivo mapping of the human basal ganglia and thalamic connectome in individuals using 7t mri. PloS one, 7(1), e29153.

Li, J., Li, Y., Gutierrez, L., Xu, W., Wu, Y., Liu, C., .. Wei, H. (2019). Imaging the centromedian thalamic nucleus using quantitative susceptibility mapping. Front Hum Neurosci, 13, 447.

Li, X., Chen, L., Kutten, K., Ceritoglu, C., Li, Y., Kang, N., .. others (2019). Multi-atlas tool for automated segmentation of brain gray matter nuclei and quantification of their magnetic susceptibility. Neuroimage, 191, 337-349.

Lim, I.A.L., Faria, A.V., Li, X., Hsu, J.T., Airan, R.D., Mori, S., van Zijl, P.C. (2013). Human brain atlas for automated region of interest selection in quantitative susceptibility mapping: application to determine iron content in deep gray matter structures. Neuroimage, 82, 449-469.

Liu, C. (2010). Susceptibility tensor imaging. Magnetic Resonance in Medicine: An Official Journal of the International Society for Magnetic Resonance in Medicine, 63(6), 1471-1477.

Manera, A.L., Dadar, M., Fonov, V., Collins, D.L. (2019). Cerebra: Accurate registration and manual label correction of mindboggle-101 atlas for mniicbm152 template. BioRxiv.

Matsumoto, J., Fossett, T., Kim, M., Duffy, J., Strand, E., McKeon, A., ... Klassen, B. (2016). Precise stimulation location optimizes speech outcomes in essential tremor. Parkinsonism Relat Disord, 32, 60-65.

Mazziotta, J., Toga, A., Evans, A., Fox, P., Lancaster, J., Zilles, K., .. o others (2001). A probabilistic atlas and reference system for the human brain: 
International consortium for brain mapping (icbm). Philosophical Transactions of the Royal Society of London. Series B: Biological Sciences, 356 (1412), 1293-1322.

Mazziotta, J.C., Toga, A.W., Evans, A., Fox, P., Lancaster, J., et al. (1995). A probabilistic atlas of the human brain: theory and rationale for its development. Neuroimage, 2(2), 89-101.

Merkl, A., Neumann, W.-J., Huebl, J., Aust, S., Horn, A., Krauss, J.K., ... others (2016). Modulation of beta-band activity in the subgenual anterior cingulate cortex during emotional empathy in treatment-resistant depression. Cerebral Cortex, 26(6), 2626-2638.

Morel, A., Magnin, M., Jeanmonod, D. (1997). Multiarchitectonic and stereotactic atlas of the human thalamus. Journal of Comparative Neurology, $387(4), 588-630$.

Neumann, W.-J., Jha, A., Bock, A., Huebl, J., Horn, A., Schneider, G.-H., . . Kühn, A.A. (2015). Cortico-pallidal oscillatory connectivity in patients with dystonia. Brain, 138(7), 1894-1906.

Pauli, W.M., Nili, A.N., Tyszka, J.M. (2018). A high-resolution probabilistic in vivo atlas of human subcortical brain nuclei. Scientific data, 5(1), $1-13$.

Peterson, E.T., Kwon, D., Luna, B., Larsen, B., Prouty, D., De Bellis, M.D., ... others (2019). Distribution of brain iron accrual in adolescence: Evidence from cross-sectional and longitudinal analysis. Human brain mapping, 40(5), 1480-1495.

Ravanfar, P., Loi, S.M., Syeda, W.T., Van Rheenen, T.E., Bush, A.I., Desmond, P., ... Pantelis, C. (2021). Systematic review: Quantitative susceptibility mapping (qsm) of brain iron profile in neurodegenerative diseases. Frontiers in Neuroscience, 15, 41. Retrieved from https://www.frontiersin.org/article/10.3389/fnins.2021.618435

\subsection{9/fnins.2021.618435}

Rolls, E.T., Huang, C.-C., Lin, C.-P., Feng, J., Joliot, M. (2020). Automated anatomical labelling atlas 3. NeuroImage, 206, 116189. 
Schweser, F., Deistung, A., Lehr, B.W., Reichenbach, J.R. (2011). Quantitative imaging of intrinsic magnetic tissue properties using mri signal phase: an approach to in vivo brain iron metabolism? Neuroimage, 54 (4), 2789-2807.

Schweser, F., Martins, A.L.R.D., Hagemeier, J., Lin, F., Hanspach, J., Weinstock-Guttman, B., ... Zivadinov, R. (2018). Mapping of thalamic magnetic susceptibility in multiple sclerosis indicates decreasing iron with disease duration: a proposed mechanistic relationship between inflammation and oligodendrocyte vitality. Neuroimage, 167, 438-452.

Shmueli, K., de Zwart, J.A., van Gelderen, P., Li, T.-Q., Dodd, S.J., Duyn, J.H. (2009). Magnetic susceptibility mapping of brain tissue in vivo using mri phase data. Magnetic Resonance in Medicine: An Official Journal of the International Society for Magnetic Resonance in Medicine, 62(6), $1510-1522$.

Smith, S.M. (2002). Fast robust automated brain extraction. Human brain mapping, 17(3), 143-155.

Starr, P.A., Christine, C.W., Theodosopoulos, P.V., Lindsey, N., Byrd, D., Mosley, A., Marks, W.J. (2002). Implantation of deep brain stimulators into subthalmic nucleus: technical approach and magnetic imaging - verified electrode locations. Journal of neurosurgery, 97(2), $370-387$.

Tustison, N.J., Avants, B.B., Cook, P.A., Zheng, Y., Egan, A., Yushkevich, P.A., Gee, J.C. (2010). N4itk: improved n3 bias correction. IEEE transactions on medical imaging, 29(6), 1310-1320.

Voges, J., Volkmann, J., Allert, N., Lehrke, R., Koulousakis, A., Freund, H.-J., Sturm, V. (2002). Bilateral high-frequency stimulation in the subthalamic nucleus for the treatment of parkinson disease: correlation of therapeutic effect with anatomical electrode position. Journal of neurosurgery, 96(2), 269-279. 
Wang, H., Suh, J.W., Das, S.R., Pluta, J.B., Craige, C., Yushkevich, P.A. (2012). Multi-atlas segmentation with joint label fusion. IEEE transactions on pattern analysis and machine intelligence, 35(3), 611-623.

Ward, P.G., Harding, I.H., Close, T.G., Corben, L.A., Delatycki, M.B., Storey, E., ... Egan, G.F. (2019). Longitudinal evaluation of iron concentration and atrophy in the dentate nuclei in friedreich ataxia. Movement Disorders, 34(3), 335-343.

Wei, H., Dibb, R., Zhou, Y., Sun, Y., Xu, J., Wang, N., Liu, C. (2015). Streaking artifact reduction for quantitative susceptibility mapping of sources with large dynamic range. NMR in Biomedicine, 28(10), 12941303.

Wharton, S., Schäfer, A., Bowtell, R. (2010). Susceptibility mapping in the human brain using threshold-based k-space division. Magnetic resonance in medicine, 63(5), 1292-1304.

Williams, N., \& Okun, M. (2013). Deep brain stimulation (dbs) at the interface of neurology and psychiatry. J Clin Invest, 123(11), 4546-56.

Winkler, A.M., Ridgway, G.R., Webster, M.A., Smith, S.M., Nichols, T.E. (2014). Permutation inference for the general linear model. NeuroImage, 92, 381-397. Retrieved from https://www.sciencedirect.com/science/article/pii/S1053811914000913

https://doi.org/10.1016/j.neuroimage.2014.01.060

Woolrich, M.W., Jbabdi, S., Patenaude, B., Chappell, M., Makni, S., Behrens, T., ... Smith, S.M. (2009). Bayesian analysis of neuroimaging data in fsl. Neuroimage, 45(1), S173-S186.

Wu, B., Li, W., Guidon, A., Liu, C. (2012). Whole brain susceptibility mapping using compressed sensing. Magnetic resonance in medicine, 67(1), 137147.

Xiao, Y., Bailey, L., Chakravarty, M.M., Beriault, S., Sadikot, A.F., Pike, G.B., Collins, D.L. (2012). Atlas-based segmentation of the subthalamic nucleus, red nucleus, and substantia nigra for deep brain 
stimulation by incorporating multiple mri contrasts. International conference on information processing in computer-assisted interventions (pp. 135-145).

Yushkevich, P.A., Piven, J., Cody Hazlett, H., Gimpel Smith, R., Ho, S., Gee, J.C., Gerig, G. (2006). User-guided 3D active contour segmentation of anatomical structures: Significantly improved efficiency and reliability. Neuroimage, 31 (3), 1116-1128.

Zhang, Y., Wei, H., Cronin, M.J., He, N., Yan, F., Liu, C. (2018). Longitudinal atlas for normative human brain development and aging over the lifespan using quantitative susceptibility mapping. NeuroImage, 171, 176-189.

Zucca, F., Bellei, C., Giannelli, S., Terreni, M., Gallorini, M., Rizzio, E., ... Zecca, L. (2006). Neuromelanin and iron in human locus coeruleus and substantia nigra during aging: consequences for neuronal vulnerability. Journal of neural transmission, 113(6), 757-767. 


\section{Appendices}

Table 2: The names and the displayed colors of the total 64 thalamic nuclei.

\begin{tabular}{|c|c|c|}
\hline No. & Name & Color \\
\hline 1 & R-pf(parafascicularis thalami) & \\
\hline 2 & L-pf(parafascicularis thalami) & \\
\hline 3 & R-pc(paracentral) & \\
\hline 4 & L-pc(paracentral) & \\
\hline 5 & $\mathrm{R}-\mathrm{Ce}$ (centralis thalami) & \\
\hline 6 & $\mathrm{~L}-\mathrm{Ce}($ centralis thalami) & \\
\hline 7 & R-Pu.l(Pulvinar thalami laterale) & \\
\hline 8 & L-Pu.l(Pulvinar thalami laterale) & \\
\hline 9 & R-Pu.m(Pulvinar thalami mediale) & \\
\hline 10 & L-Pu.m(Pulvinar thalami mediale) & \\
\hline 11 & R-Pu.o.v(Pulvinar thalami orale-ventralis) & \\
\hline 12 & L-Pu.o.v(Pulvinar thalami orale-ventralis) & \\
\hline 13 & R-V.c(ventrocaudalis) & \\
\hline 14 & L-V.c(ventrocaudalis) & \\
\hline 15 & R-Li(limitans thalami) & \\
\hline 16 & L-Li(limitans thalami) & \\
\hline 17 & R-V.i.m(ventrointermedius) & \\
\hline 18 & L-V.i.m(ventrointermedius) & \\
\hline 19 & R-V.o.p(ventrooralis posterior) & \\
\hline 20 & L-V.o.p(ventrooralis posterior) & \\
\hline 21 & R-V.o.a(ventrooralis anterior) & \\
\hline 22 & L-V.o.a(ventrooralis anterior) & \\
\hline 23 & R-V.o.i(ventrooralis internus) & \\
\hline 24 & L-V.o.i(ventrooralis internus) & \\
\hline 25 & R-Lpo(lateropolaris thalami) & \\
\hline 26 & L-Lpo(lateropolaris thalami) & \\
\hline 27 & $\mathrm{R}-\mathrm{Fa}$ (fasciculosus thalami) & \\
\hline 28 & $\mathrm{~L}-\mathrm{Fa}$ (fasciculosus thalami) & \\
\hline 29 & R-T.mth(Tractusmammillo-thalamicus) & \\
\hline 30 & L-T.mth(Tractusmammillo-thalamicus) & \\
\hline 31 & $\mathrm{R}$-mc in Lpo & \\
\hline 32 & L-mc in Lpo & \\
\hline 33 & R-La.m.o(lamella medialis oralis) & \\
\hline 34 & L-La.m.o(lamella medialis oralis) & \\
\hline 35 & R-S.pv(Substantia periventricularis) & \\
\hline 36 & L-S.pv(Substantia periventricularis) & \\
\hline 37 & $\mathrm{R}$-mc in $\mathrm{Ce}$ & \\
\hline 38 & L-mc in $\mathrm{Ce}$ & \\
\hline \multicolumn{3}{|c|}{ Continued on next page } \\
\hline
\end{tabular}


Table 2 - continued from previous page

\begin{tabular}{|c|l|l|}
\hline No. & \multicolumn{1}{|c|}{ Name } & Color \\
\hline 39 & R-F.M(fasciculus retroflexus Meynertii) & \\
40 & L-F.M(fasciculus retroflexus Meynertii) & \\
41 & R-V.o.m(ventrooralismedialis) & \\
42 & L-V.o.m(ventrooralismedialis) & \\
43 & R-Pu.ol(Pulvinar thalami oral laterale) & \\
44 & L-Pu.ol(Pulvinar thalami oral laterale) & \\
45 & R-Pu.om(Pulvinar thalami orale mediale) & \\
46 & L-Pu.om(Pulvinar thalami orale mediale) & \\
47 & R-Pu.sf(pulvinaris superficialis) & \\
48 & L-Pu.sf(pulvinaris superficialis) & \\
49 & R-D.c(dorso-caudalis) & \\
50 & L-D.c(dorso-caudalis) \\
51 & R-D.im(dorso-intermedii) \\
52 & L-D.im(dorso-intermedii) \\
53 & R-D.o(dorso-orales thalami) \\
54 & L-D.o(dorso-orales thalami) \\
55 & R-M(dorsomedialis) \\
56 & L-M(dorsomedialis) \\
57 & R-A.pr(anteroprincipalis thalami) & \\
58 & L-A.pr(anteroprincipalis thalami) & \\
59 & R-CL(centralis lateralis) \\
60 & L-CL(centralis lateralis) \\
61 & R-La.m.ip(lamella medialis interpolaris) & \\
62 & L-La.m.ip(lamella medialis interpolaris) \\
63 & R-LMM(Lamina medullaris medialis) \\
64 & L-LMM(Lamina medullaris medialis) & \\
\hline
\end{tabular}

